\title{
1 Disordered information processing dynamics in experimental epilepsy
}

2 Wesley Clawson ${ }^{1}$, Tanguy Madec ${ }^{1}$, Antoine Ghestem ${ }^{1}$, Pascale P Quilichini, ${ }^{1, *}$, Demian

3 Battaglia ${ }^{1, *}$, Christophe Bernard ${ }^{1, *}$

4 1. Aix Marseille Univ, Inserm, INS, Institut de Neurosciences des Systèmes, Marseille, France

$5 \quad *$ - Equally contributing last authors

7 Abstract

8 Neurological disorders share common high-level alterations, such as cognitive deficits, anxiety,

9 and depression. This raises the possibility of fundamental alterations in the way information

10 conveyed by neural firing is maintained and dispatched in the diseased brain. Using

11 experimental epilepsy as a model of neurological disorder we tested the hypothesis of altered

12 information processing, analyzing how neurons in the hippocampus and the entorhinal cortex

13 store and exchange information during slow and theta oscillations. We equate the storage and

14 sharing of information to low level, or primitive, information processing at the algorithmic level,

15 the theoretical intermediate level between structure and function. We find that these low-level

16 processes are organized into substates during brain states marked by theta and slow

17 oscillations. Their internal composition and organization through time are disrupted in epilepsy,

18 loosing brain state-specificity, and shifting towards a regime of disorder in a brain region

19 dependent manner. We propose that the alteration of information processing at an algorithmic

20 level may be a mechanism behind the emergent and widespread co-morbidities associated with

21 epilepsy, and perhaps other disorders. 
Introduction

23 Most, if not all, neurological pathologies, including Alzheimer's disease, epilepsies, and

24 Parkinson's disease, aside from their specificities, display commonalities in terms of cognitive

25 (e.g., memory) and mental (e.g., anxiety and depression) disorders (Hesdorffer, 2016).

26 Historically, attempts have been made to correlate higher-level changes to the underlying

27 structural alterations. However, structural alterations may be very different from one pathology

28 to the next, even within a given brain disorder. The origin of shared and generic deficits must

29 therefore be sought for at a level higher than the structural one. We hypothesize that diverse

30 pathological mechanisms can lead to similar modifications of information processing, emerging

31 from, and existing between, structural and functional levels. Whether information processing is

32 modified in a pathological context is not known. Furthermore, a formal framework for the

33 quantification of these processes is missing.

35 As a model of neurological disorder, we consider Temporal Lobe Epilepsy (TLE), the most

36 common form of epilepsy in adults (Tatum, 2012). TLE is itself highly heterogenous in terms of

37 differences of histopathology (Blumcke et al., 2013), semiology (Barba et al., 2007; Bartolomei et

38 al., 2008) and cognition and mental state (de Barros Lourenco et al., 2020; Holmes, 2015;

39 Krishnan, 2020). Such heterogeneity is also found in experimental models of TLE (Rusina et al.,

40 2021). Structural alterations may change several features that are relevant for information

41 processing, such as rate coding, temporal coding, synaptic plasticity, and network oscillations

42 (Lenck-Santini \& Scott, 2015). In keeping with this proposal, hippocampal place cells are unstable,

43 firing becomes randomized during ripples, synaptic plasticity, and oscillations are altered, and 
44 these changes are correlated with deficits in hippocampus-dependent spatial memory in

45 experimental epilepsy (Chauvière et al., 2009; Inostroza et al., 2013; Lenck-Santini \& Holmes,

46 2008; Lopez-Pigozzi et al., 2016; Suarez et al., 2012; Valero et al., 2017). Given this diversity of

47 deficits, it is reasonable to presume that in TLE local information processing is altered at a more

48 fundamental level, with widespread impacts on multiple functions.

50 It is difficult to link specific alterations at the structural level to high order cognitive deficits as we

51 do not know where information processing is localized, what is being processed, nor how it is

52 integrated into function. In other words, with reference to the notion of the algorithmic level

53 introduced by Marr and Poggio (1977), we do not know what are the "algorithms" that bridge

54 structure and function. The common axiomatic view is that neural information processing stems

55 from the spatiotemporal organization of the firing of neurons. Information theory was designed

56 to be agnostic to the content of information and thus provides useful metrics to track primitive,

57 or fundamental, information processing operations (Shannon, 1948). Neuronal firing intrinsically

58 carries information due to its statistical properties. Auto-correlations in firing actively maintain

59 this information through time - active information storage (Lizier et al., 2012; Wibral et al., 2014),

60 and cross-correlated firing between different neurons allows the sharing of this information

61 between themselves (Kirst et al., 2016). Focusing on such basic operations allows investigation

62 of how patterns of coordinated neural firing may translate into primitive low-level information

63 processing (Clawson et al., 2019), akin to the algorithmic level. Here, we hypothesize that the key

64 differences between control and epileptic networks are not only present at the structural level,

65 but also at a more general and core algorithmic level of quantifiable primitive operations. 
67 To test this hypothesis, a multilevel experimental approach is required (Scott et al., 2018). Multi-

68 channel electrode recordings of neural populations provide such a dataset which spans two levels

69 of analysis: the action potential at the neuronal level and oscillations at the population level. As

70 neural computation is brain state dependent (Quilichini \& Bernard, 2012), we consider the global

71 brain states of theta (THE) and slow oscillations (SO), which can be recorded during anesthesia.

72 Previous work in control animals demonstrate that neuronal activity patterns in the hippocampus

73 and entorhinal cortex switch between different information processing substates (IPSs) (Clawson

74 et al., 2019). An IPS corresponds to an epoch in which primitive operations of information storage

75 and sharing in a local microcircuit remain temporally consistent. IPSs continuously switch from

76 one IPS to another, similarly to what has been described at higher level of organization, such as

77 the dynamics of resting state networks and EEG microstates (Calhoun et al., 2014; Van de Ville et

78 al., 2010). In the control hippocampus and entorhinal cortex, the sequences of IPSs are complex,

79 i.e. standing between order and disorder (Clawson et al., 2019).

81 Using an unbiased quantification of IPSs, we compare their properties and organization between

82 control and experimental epilepsy conditions. We focus on the hippocampus and the entorhinal

83 cortex, two major structures commonly affected in TLE (Curia et al., 2008). We find that IPS'

84 internal organization and switching dynamics, although not suppressed, shift toward a less

85 structured and more random spatiotemporal organization in experimental epilepsy than in

86 control. Such disruption of information processing at the algorithmic level itself could underly the

87 general performance impairments in TLE. 
Results

Design

90 We analyze the local field potentials (LFPs) and action potentials from individual neurons

91 measured in the hippocampus (CA1) and medial entorhinal cortex $(m E C)$ from control $(n=5)$ and

92 experimental epilepsy $(n=6)$ rats under anesthesia (Figure $1 A-B$, see Methods for details).

93 Unsupervised clustering of the spectral content of LFPs reveals that field activity continuously

94 switches between two states: slow oscillations (SO, 0.5-3 Hz) and theta oscillations (THE, 3-6 Hz)

95 (Figure 1B, S1). As previously reported in freely moving animals (Chauvière et al., 2009), THE

96 power and peak frequency are decreased in CA1 in experimental epilepsy (Figure S1). Although,

97 the peak frequencies of THE and SO are not modified in the MEC in epilepsy, their power is

98 decreased (Figure S1). However, both frequency and power ratios between SO and THE are

99 similar in control and epilepsy.

100

101 We extract three features from the spike trains using a sliding widow procedure (Figure 1B-C):

102 (1) firing, the number of times a neuron fired within a window, (2) storage, the information

103 theoretical measure of active information storage (Lizier et al., 2012; Wibral et al., 2014), which

104 captures temporal patterns of spiking for a single neuron within a window - notably in our case,

105 how regular or repetitive these patterns are - and (3) sharing, an information theoretical

106 measure of information sharing (Kirst et al., 2016), which captures spatiotemporal patterns of

107 coordinated spiking across neurons within a window. First, we examine whether these features

108 are dependent upon the brain state (THE versus SO), the region (CA1 versus mEC) and the 109 condition (control versus epilepsy). 


\section{Epilepsy reduces firing, storage and sharing differences between THE and SO states}

111 In control animals, we find that in both regions, average firing and storage of all neurons is larger

112 during THE than SO, while average sharing is lower (Figure 1D, see also S2), in keeping with the

113 idea that neuronal computation is brain state-dependent (Quilichini \& Bernard, 2012). In

114 epilepsy, we find that average firing and storage are decreased during THE, but not during SO, as

115 compared to control in both $\mathrm{MEC}$ and HPC. As a consequence, the brain state-dependency of

116 firing and storage, which is consistent across controls, is reduced in both regions in epilepsy

117 (Figure 1D). There is thus, in epilepsy, a large deviation from the operating mode found in control

118 conditions.

120 We have previously shown that THE and SO states are in fact characterized by a complex dynamic

121 organization in terms of firing, storage or sharing features (Clawson et al., 2019). A feature value

122 (e.g., storage) can remain stable during a given time period (i.e., during successive windows),

123 before switching to a different feature value with its own period of stability. We called these

124 periods of stability substates of firing, storage or sharing. We begin by assessing the properties

125 of substates in control and in epilepsy, as substate switching constitutes an important qualitative 126 aspect of coordinated firing dynamics. 
A

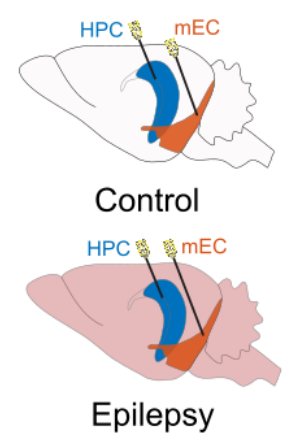

B

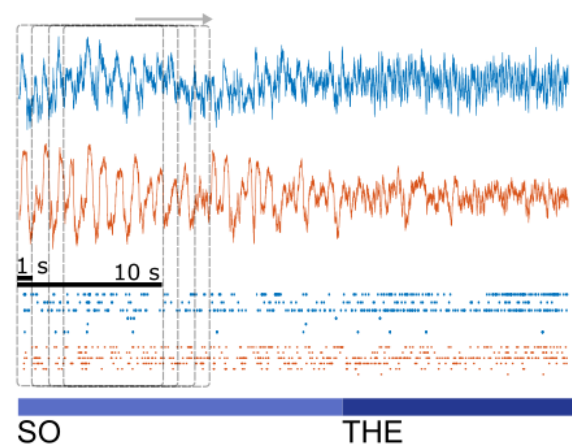

C Feature Vectors
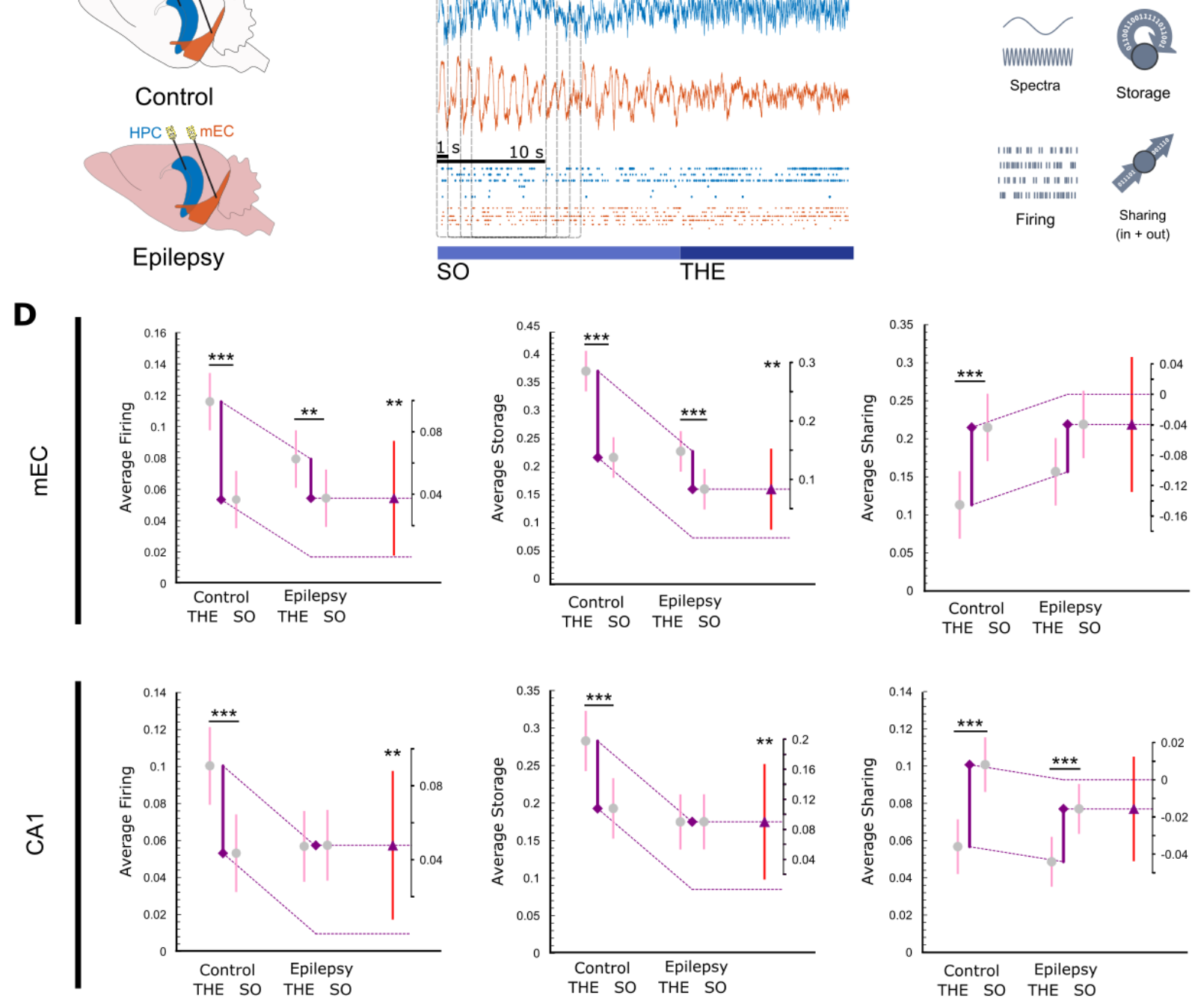

130 Figure 1: Experimental and analytical design - (A) Cartoon representing the approximate recording

131 locations in $\mathrm{mEC}$ (orange) and CA1 (blue) in control and experimental epilepsy. (B) Example of LFP (top)

132 and firing (bottom, each line represents one neuron, a dot represents an action potential) data recorded

133 in control CA1 and MEC during SO and THE. Overlayed is a representation of our analytic method that

134 uses $10 \mathrm{~s}$ long sliding windows shifted by $1 \mathrm{~s}$ at each step. (C) Cartoon examples of the four acquired

135 data features. (D) Average values and difference of differences graphs for data features taken from

136 spiking data during epochs of THE and SO in mEC (top) and CA1 (bottom) in both control and epilepsy 
137 conditions. See S2 for the same graph represented as a function of region, rather than oscillatory state.

138 Circles and triangles represent the mean, and all bars represent a 99\% bootstrapped confidence interval.

139 Significance is shown using the symbol $(*)$ with their standard corresponding meaning $(*, p<0.05 ; * *$,

$140 \mathrm{p}<0.01 ; * * *, \mathrm{p}<0.001)$. The numerical values are provided in Table S1.

\section{Terminology, metrics, and methodology}

143 Figure $2 \mathrm{~A}$ illustrates an example of the procedure for a $25 \mathrm{~min}$ long recording performed in the

144 mEC in a control animal. Spectral analysis of the LFP reveals the alternation between THE and SO

145 states (upper row). Through an unsupervised substate extraction procedure based on $k$-means

146 clustering (see Methods), we identify in this example 4, 3, and 5 substates of stable patterns for

147 firing, storage and sharing, respectively. The four features together, seen as 4 rows in Fig 2A,

148 define a switching table. Each time point in the table corresponds to an information processing

149 state (IPS), i.e. a combination of global state, firing rate, storage, and sharing patterns at this time

150 point. By characterizing which neurons fire, how much, and with which correlation properties, an

151 IPS provides a robust characterization of the pattern of coordinated activity occurring within each

152 temporal window. Note that the switching transitions from one substate to the next are not

153 necessarily synchronous between the different features, a property found in all recordings. In

154 Figure 2B, we show, encoded as vertical color bars, the absolute values of firing, storage and

155 sharing features that different neurons assume in the different substates. For a given feature,

156 the values appear clearly different for a given neuron between substates. We will quantify these

157 differences in the next section. 
159 The switching table of Figure $2 \mathrm{~A}$ is constructed using an unsupervised clustering algorithm, $\mathrm{k}-$

160 means, guided by an a priori assumption that (1) there exist separable clusters of data and (2)

161 there are exactly $k$ of these clusters (here 4, 3, and 5 for firing, storage and sharing, respectively).

162 Using a null model, we demonstrate that there exist separable clusters (Figure S3). However, as

163 the ground truth of how many clusters exist is unknown, statistical criteria can be used to find

164 the optimal number (as done in Clawson et al., 2019). Here, we use a more general approach

165 varying the $k$ value for each firing, storage, and sharing feature while fixing $k=2$ for the spectral

166 feature. Each quadruplet of $k$ values will produce a specific switching table. Figure $2 \mathrm{C}$ illustrates

167 this concept, showing the resultant clustering of storage substates through time as $k$ increases

168 from 3 to 10. A low value may underestimate the real number of substates, while a large number

169 may be an overestimate producing substates that rarely occur more than once (see Methods).

170 We therefore use a lower bound of $k=3$, and a reasonable upper bound of $k=10$, wherein the

171 clusters become too fine (Figure 2C, see Methods). We thus consider eight possible $k$ values for

172 each feature, giving rise to $8^{3}=512$ possible switching tables. Each switching table is

173 characterized by the total number of substates it contains: $k_{\text {tot }}=2+k_{\text {firing }}+k_{\text {storage }}+k_{\text {sharing }}$ with a

174 maximum value of $k_{\max }=32(32=2$, the number of spectral states +3 features $\times 10)$. The

175 collection of all switching tables for a given recording defines a library of tables (Figure 2D). We

176 chose such a method with the intention that without an a priori approach on the underlying

177 principle, if we extract generic rules, they should be valid independently of the choice of number

178 of clusters, at least for a reasonable wide range of $k$ values. Now, all analysis that can be done on

179 a switching table is performed for each library, which gives the added benefit of assessing the

180 robustness of the results regarding the number of clusters. 


\section{Substates are more contrasted in epilepsy}

183 The vertical color bars in Figure 2B qualitatively show that individual neurons can take different

184 firing, storage or sharing values across substates. In order to quantify these differences, we 185 measure how "contrasted" are different substates. If we consider the firing feature of a given 186 neuron, we first calculate its global mean firing rate (over the whole duration of the recording), 187 and its mean firing rate within each substate. The relative contrast is defined as the difference 188 between the substate mean firing rate and the global mean firing rate, normalized by the global 189 mean firing rate. Evaluating contrast allows better tracking of the differing compositions of

190 substates at the single neuron level. Figure 2B shows the relative contrast plots for the 44

191 recorded neurons and the various substates in the same dataset and substate decomposition we

192 use as an example in Figure 2A. The differences between substates for each feature now clearly 193 appear as large changes in the distributions of contrast values for the recorded neurons. Now,

194 we extract the substate contrast of each substate for each feature - the average of the absolute 195 values of the heights of the bars in the relative contrast plot. This substate contrast tells us how 196 much a given substate stands out from its feature's global average. Increasing the number of $k$ 197 substates may decrease the substate contrast.

199 Figure 2E shows the distributions of the differences in contrasts between control $(n=5)$ and 200 epilepsy $(n=6)$, for firing, storage, and sharing features in the $\mathrm{mEC}$, for the chosen $k$ values 201 ( $\left.3 \leq k_{\text {firing }}, k_{\text {storage, }} k_{\text {sharing }} \leq 10\right)$. For all values of $k$, for all features, the contrast differences lie 202 entirely below zero, demonstrating that substate contrast is generally higher in epilepsy than in 
203 control. We also see no clear dependence upon $k$ values, i.e., the number of substates. The same

204 result is found in CA1, however higher bounds closer to the $99^{\text {th }}$ percentile do cross 0 (Fig S4).

205 We thus identify another major alteration in epilepsy; substates are more contrasted, exhibiting

206 more marked differences with respect to the mean. This suggest that in epilepsy, substate

207 switching more strongly modulates the neural population with regards to firing, storage and

208 sharing. While this seems to stand in contrast with the previously described reduction of the

209 modulatory influence exerted by global oscillatory states, this may be explained by a disrupted

210 articulation between substate and global state, as we explain in the following section. 
A

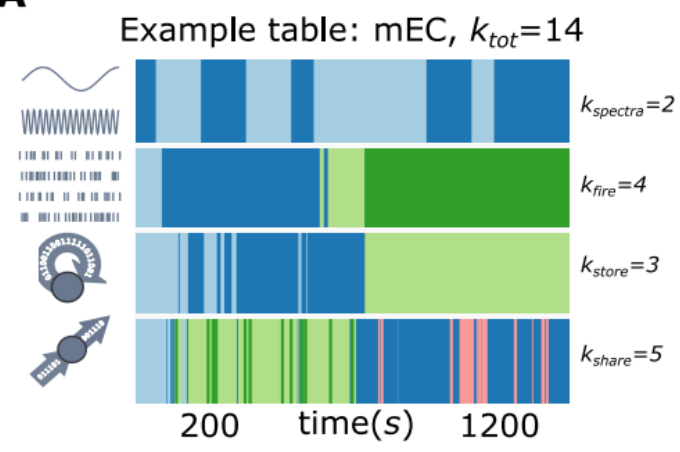

B

范

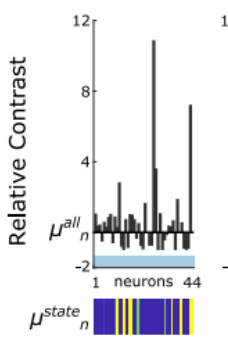

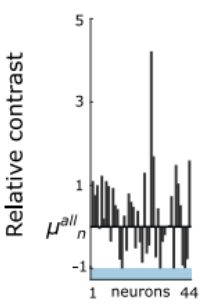
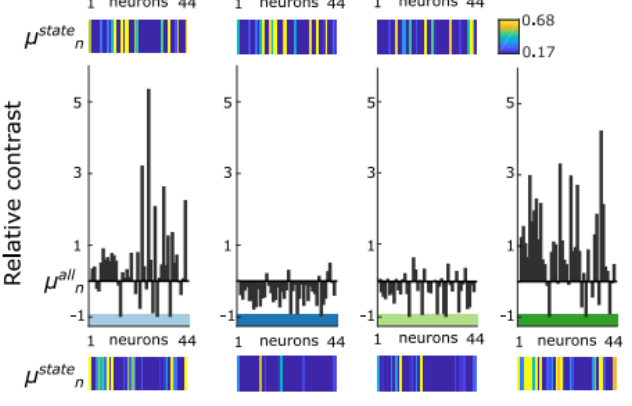

C

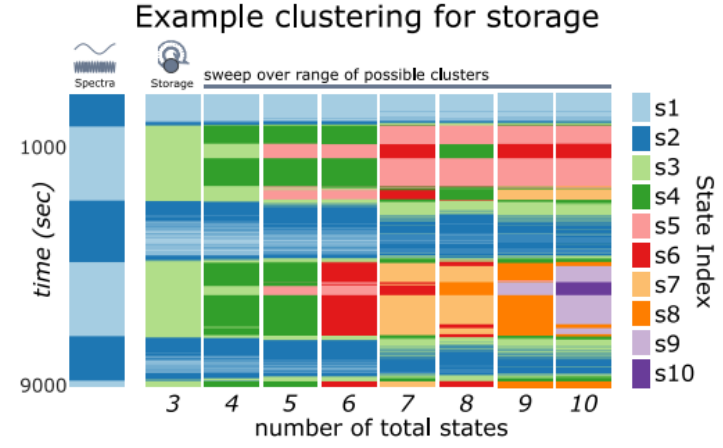

D

Library of State Tables

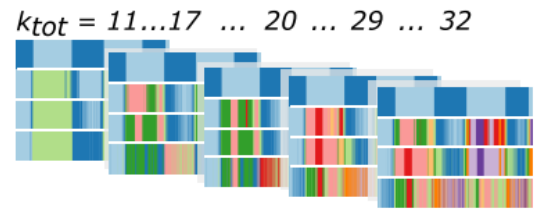

$\mathbf{E}$

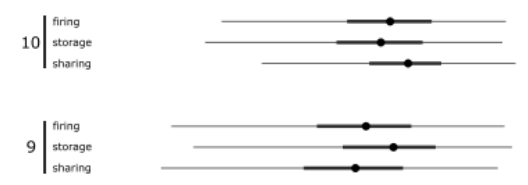

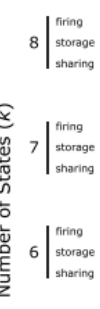

$\left.5\right|^{\begin{array}{l}\text { firing } \\ \text { storage } \\ \text { sharing }\end{array}}$

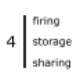

$\left.3\right|_{\substack{\text { fring } \\ \text { storage } \\ \text { sharing }}} ^{\text {sher }}$
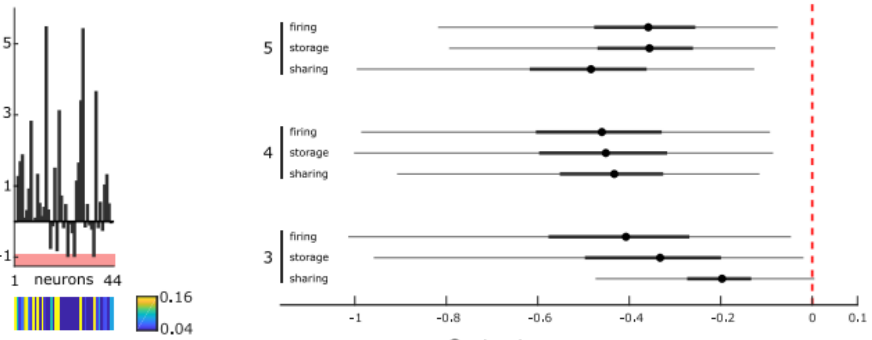

Contrast, $\mu_{\text {control }}-\mu_{\text {epilepsy }}$

212 Figure 2 - Clustering \& contrast in control and epilepsy - (A) An example state table for the mEC

213 in a control animal with a total state count of $k_{t o t}=14$. The different substates are color coded.

214 Note that switching is not synchronized across the different features. (B) Relative contrast values

215 for the table given shown in (A). The substates shown in A are shown in B as a horizontal bar with

216 the same color. Each graph shows the relative contrast of each of the 44 neurons, for each

217 substate, and each feature. Below each graph is a visual indicator of a neuron's feature values 
218 within the substate (vertical color bar). Here, the color scale varies from near 0 , dark blue, to the

219 top $10 \%$ of all average activity within the state. Therefore, any neuron whose activity is within

220 this top $10 \%$ will be bright yellow. (C) Temporal dynamics (vertical axis) of storage substates as a

221 function of $k$ (horizontal axis). The far-left column shows the dynamics of THE and SO spectral

222 states. (D) An example of a resulting state table library, or a collection of all possible combinations

223 of all clustering with a range of $k_{t o t}=11-32$. (E) Average contrast difference between control

224 and epilepsy is shown with respect to both feature and number of states, $k$. The circles represent

225 the mean difference, the thick black bars represent the $25-75 \%$ quantile and the thin black bars

226 represent the $1-99 \%$ quantile. The red dotted line is to add the null hypothesis line of no

227 significant difference between control and epilepsy.

Loss of global state specificity of firing, sharing and storage substates in epilepsy

230 Since firing patterns are brain state-dependent, we assess whether this type of specificity is also

231 found at the level of information processing substates. For a given state table in a library, we

232 calculate the probability that a substate occurs during THE, SO or both. We name it state

233 specificity index (SSI), a metric bounded between 0 (a substate occurs equally in THE or SO) and

2341 (a substate is exclusive to either THE or SO) (see Methods). In control animals (Figure 3, blue

235 curves), most substates are brain state specific in both mEC and CA1, independently of $k$. Most

236 SSI values are above 0.8 , well above the null hypothesis $0.23 \pm 0.03$ value of lack of global state

237 specificity. Global state specificity of substates is thus a robust result in control animals with 238 respect to $k$. 
239 The same analysis performed in epilepsy reveals a region dependent alteration in SSI (Figure 3,

240 red curves). There is a large decrease in SSI for all features in the mEC, indicating a loss of the

241 constraint exerted by global oscillatory states on the selection of possible substates, again

242 regardless to the chosen $k^{\prime}$ s. In contrast, there is no such large loss of brain state specificity in

243 CA1, in particular no change for sharing. We conclude that the substate distribution becomes

244 "disordered", i.e., a large proportion of substates now occur during both THE and SO in the mEC

245 in epilepsy. In contrast, CA1 retains the brain state specificity of the distribution of substates. The

246 alteration of brain state-specificity of firing, sharing and storage substates is therefore brain

247 region dependent in epilepsy. 

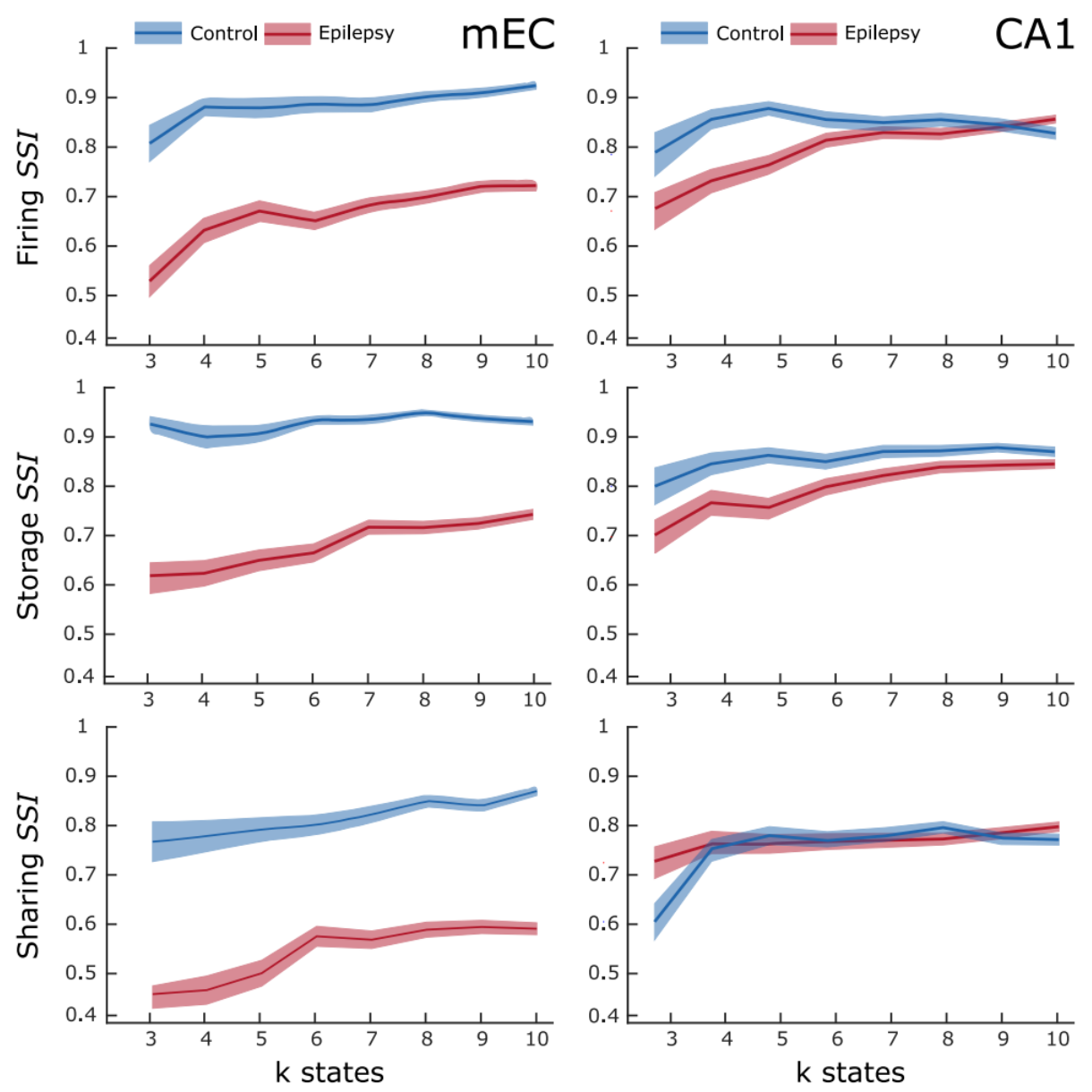

250 Figure 3 - Loss of brain state-dependency of substates in the mEC in epilepsy - State similarity

251 index (SSI) is shown here vs number of $k$ states for each feature in mEC and CA1. Blue represents

252 the control data while red represents epilepsy. The bold lines represent the mean while the

253 shaded regions represent a 99\% bootstrapped confidence interval. The bootstrapped null model

254 produced via randomizing gives an average SSI of $0.23 \pm 0.03$ and is not shown here to increase

255 visual clarity. 
Computing hubs are more numerous but less substate-specific in the mEC in epilepsy

258 Within each substate/feature we extract computing hub neurons, i.e., neurons with on average,

259 exceptionally high firing, storage or sharing values with regard to the substate (see Methods). As

260 previously discussed in Clawson et al. (2019), it is important to stress that different substates are

261 associated to different sets of hubs and that a neuron acting as firing, storage or sharing hub in a

262 given substate will not necessarily do so in another substate. So, while the fraction of neurons

263 being hub in a given substate remains small, the fraction of neurons serving as hub at least in one

264 substate is much larger, approaching $\sim 40 \%$ on average. Figure $4 \mathrm{~A}$ illustrates an example of the

265 distribution of hubs (same recording as in Figure 2A).

267 In control animals, the percentage of hubs increases with $k_{t o t}$ in both mEC and CA1 (Figure 4B),

268 which is expected due to the arbitrary over-clustering as $k$ increases. We observe furthermore

269 that the percentage of neurons serving as hubs at least once is significantly increased in epilepsy,

270 by $5 \%$ in the $\mathrm{mEC}$ and $2.5 \%$ in CA1 (Figure 4B). This result is in agreement with the increase in

271 substate contrast found in epilepsy: more neurons are more contrasted and therefore are

272 detected as hubs. Note that, for both control and epilepsy, the percentage of neurons marked as

273 hubs is significantly larger as compared to randomized state tables (grey dotted lines in Figure

274 4B), confirming that the emergence of hubs is a direct fingerprint of the existence of well distinct

275 substates. 
277 Figure 4A also shows that some computing hubs are shared by different substates, while others

278 are specific to one substate/one feature. In order to assess how substate-specific the computing

279 hubs are, we use a measure of similarity (see Methods). A null value indicates that every substate

280 has a unique hub set with no overlap between substates while a 1 value means that all substates

281 have an identical distribution. Figure 4C shows that, in control animals, a majority of hubs tend

282 to be substate-specific (similarity < 0.5). In CA1, the distribution of hubs is less substate-specific

283 than in the mEC (higher similarity). In epilepsy, the distribution of hubs does not change in CA1,

284 while hubs become significantly more substate-specific in the $\mathrm{mEC}$. In other words, the status of

285 being hub is for a mEC neuron less stable in epilepsy than in control animals.

287 We conclude that, in epilepsy, the mEC and CA1 display an increase in the number of neurons

288 labeled as hubs at least once, and that the substate-specificity of hubs is increased in the mEC.

289 Taken together, these two findings suggest a more hectic and random-like emergence of

290 computing hubs in epilepsy as compared to control, albeit expressed in different ways; in mEC

291 there are more hubs that are simultaneously more specific than control and in CA1 there are

292 more hubs while staying the same, indicating a possible 'shuffling' of hubs. We believe this also

293 further confirms that alterations in information processing are brain-region dependent. 
A

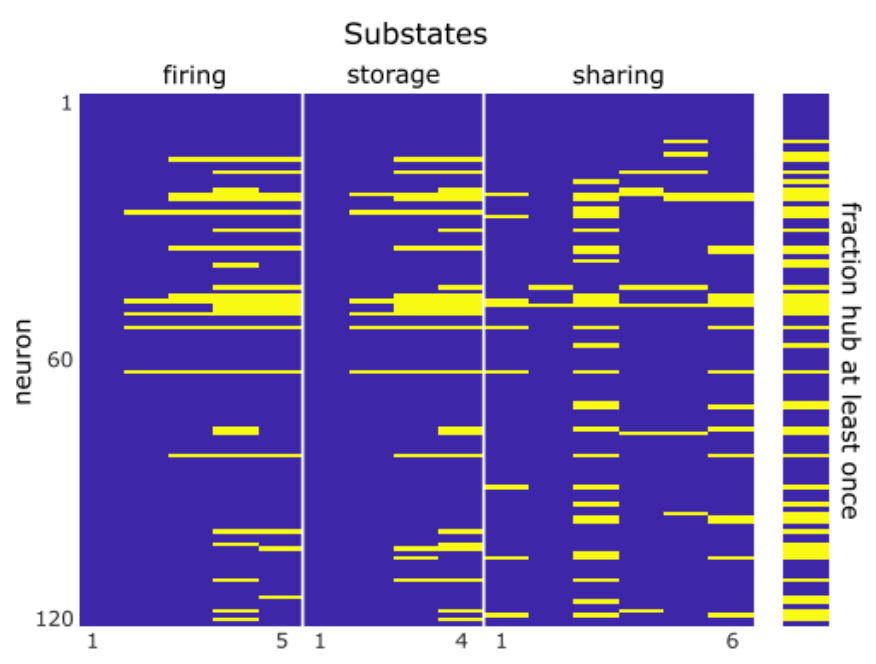

B
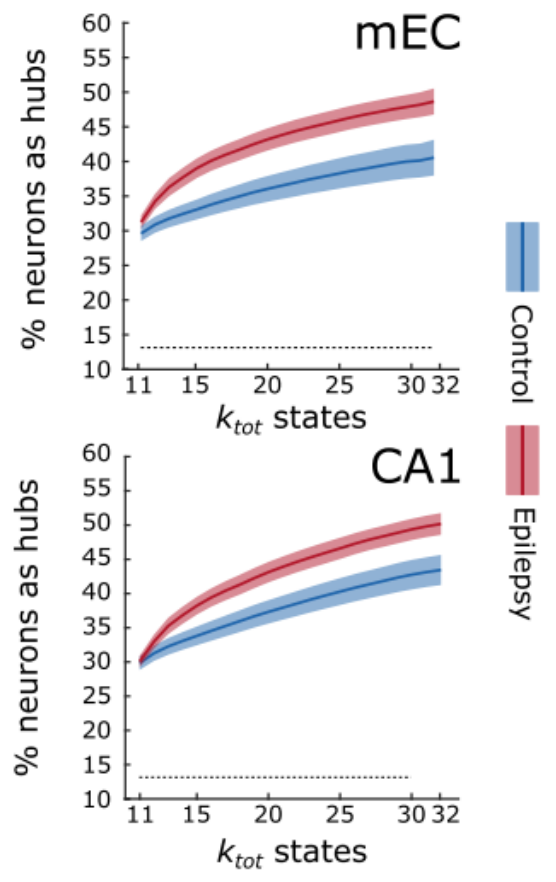
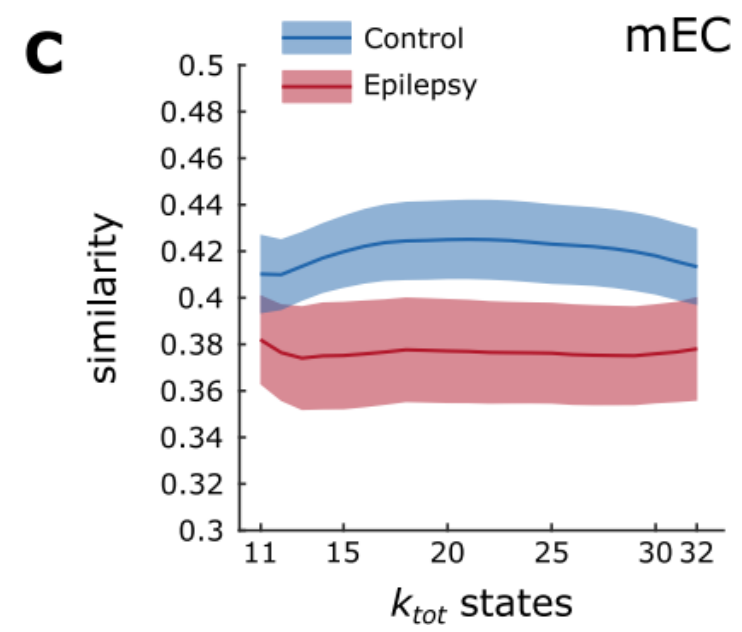

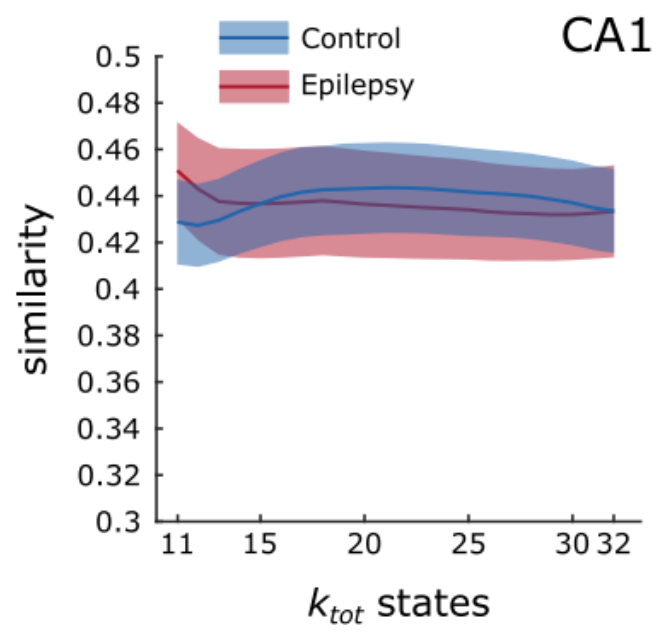

295 Figure 4 - Computing Hubs and their distributions - (A) Example of computing hubs in the control $\mathrm{mEC}$ extracted from a given state table. The $\mathrm{y}$ axis is unsorted neuron label, and the $\mathrm{x}$ axis

297 shows the substates for firing (5), storage (4) and sharing (6) features. A yellow bar indicates that

298 the given neuron is a computational hub during a substate. On the right is a summed version of

299 the graph on the left, visually showing the fraction of neurons that are a hub at least once (40\%).

300 (B) The percentage of neurons that are hubs at least once is increased in epilepsy independently 
301 of $k_{\text {tot. }}$. The grey dotted line represents the mean of the shuffled, null model. (C) The similarity

302 index plotted as a function of $k_{\text {tot. }}$. The hubs become less substate-specific in the MEC in epilepsy.

303 Blue and red are for control and epilepsy data, respectively. The bold lines are the mean, and the

304 shaded regions are the $99 \%$ bootstrapped confidence interval. 


\section{Alterations in the core-periphery organization of CA1 computing hubs in epilepsy}

307 The partners from whom a given neuron receives or to whom it sends information are 308 continuously changing (Clawson et al., 2019). At each time step, the instantaneous sharing 309 networks can be seen as having a dynamic core-periphery structure (Pedreschi et al., 2020), with

310 a core of tightly integrated neurons, surrounded by lightly connected periphery neurons. Two

311 key measures of the core-periphery structure are the coreness, how central or well-integrated

312 within a dense subnetwork - how "core"- a given neuron is, and the Jaccard index, a measure

313 indicating how similar (or, conversely, liquid) the connections are between the recorded neurons

314 between two time steps. We find that average coreness and the overall coreness distribution

315 shapes are not significantly changed in epilepsy for either mEC or CA1 (Fig S5). Thus, the core-

316 periphery architecture of information sharing networks within every substate is preserved in

317 epilepsy. However, during the SO state, the average Jaccard values in CA1 are significantly 318 decreased in epilepsy as compared to control (Fig S5). Thus, in CA1 there is enhanced connectivity 319 variance and more volatile recruitment of neurons in the core.

321 Assessing substate sequences

322 The analysis of individual features (firing, storage and sharing) revealed brain state- and brain 323 region-dependent alterations in epilepsy. We now focus on a more integrated view of the 324 informational patterns, in which we consider both the simultaneity of the ongoing types of 325 patterns and their articulation in sequences along time. We perform this higher-level exploration 326 using the notion of information processing states (IPS), driven by the idea of symbolization, as 327 shown in Figure 2A (Porta et al., 2015). From each analysis time window, we generate a four- 
328 letter word, with the letters representing the substate labels of the global state, firing, storage

329 and sharing features measured in this time window (see Methods). When the analysis window is

330 shifted by $1 \mathrm{~s}$, another word is obtained, which is identical to the previous one if the substate

331 does not change. This procedure allows us to reduce the description of the complex simultaneous

332 variations of firing, storage and sharing patterns within the neuronal population to simple strings

333 of symbolic words, a sort of "neuronal language" built of sequences of possible words in a

334 dictionary. We can then assess how the properties of these strings are modified in epilepsy at the

335 level of their dictionary and syntax.

337 We defined all possible state tables generated through our k-means procedure as a library (Fig

338 2). Now, as tables are considered as a sequence of words, we define the sequence of words

339 generated as a book. The number of letters, and therefore the number of words, depend upon

$340 k_{\text {tot. }}$ As a result, we label our differently generated books by $k_{\text {tot. }}$ All 512 possible books per

341 recording are grouped together to form a library. For each library, we build two sister libraries

342 for comparison: one in which we sort every book internally to be highly ordered, and one in which

343 we randomize every book internally to be highly disordered (see Methods). Using this word/book

344 analogy, we begin to explore the organization of the language of the information processing

345 contained in the books held within the library - What words are expressed? Is there a syntax, or

346 organizational rules? And how does epilepsy change these measures? 


\section{Impoverishment of the Dictionary in the MEC in Epilepsy}

349 For each $k_{t o t}$, there is a fixed number of potential words that can be generated and possibly

350 appear within the associated book (see cartoon in Figure 5A). As in any language, only a fraction

351 of all possible words is expressed. For each book, we measure the used dictionary fraction, or

352 relative dictionary (see Methods). Figure 5A illustrates two end cases. The low relative dictionary

353 (left) uses a small number of expressed words, while the high relative dictionary (right) uses a

354 much richer vocabulary, wherein almost all of the potential dictionary is expressed. While the

355 measure of relative dictionary in and of itself is informative, it is difficult to use such a measure

356 to assess meaningful changes (i.e., before control and epilepsy) without having comparative

357 baselines. Therefore, we compute not only the relative dictionary of our libraries, but also that

358 of the ordered and random sister libraries (which correspond to the null hypotheses of order and

359 disorder in the 'language' of the book, respectively). We then apply a linear transformation to

360 the relative dictionary measure, resulting in 0 representing the relative dictionary measure of

361 ordered books, and a value of 1 representing a relative dictionary measure identical to that of

362 randomized books. Such a normalized relative dictionary measure tracks not only the richness of

363 the used dictionary but also its position between order and disorder.

365 Figure 5B shows that for both the MEC and CA1 in control and epilepsy conditions, the normalized

366 relative dictionaries lie much closer to 0 than to 1 , meaning that their relative dictionaries are

367 much more similar to a system with organization that is ordered than disordered. In epilepsy, the

368 relative dictionary is reduced with respect to control in the mEC (Figure 5B). Thus, the dictionary

369 of state dynamics language seems impoverished in the mEC in epilepsy. There is also a reduction 
370 in CA1, but only for books with low $k_{\text {tot }}$ values whereas it is increased for $k_{\text {tot }}>15$. This 'crossing'

371 of control and epileptic near $k_{\text {tot }}=15$ may be potentially explained by the strength of clustering for sharing

372 features (Fig S3). Contrary to all features, there exists only a small window of $k$ for sharing in CA1 in which

373 k_means clusters the feature better than a null model. Therefore, dictionaries made with poor clustering

374 may drive the dictionary too high for low values of $k$. This is the first instance for which the generic

375 rule that results should be independent of the choice of k, fails. However, this characterization

376 of dictionaries further demonstrates that the alterations are brain region dependent.

378 The relative dictionary provides important information about the words, but not how words are

379 organized in time. This is similar to the grammar, or syntax, of a traditional sentence. To analyze

380 this syntax (how words are organized from one window not the next), we quantify the level of

381 organization present in the state tables as a whole, i.e., the overall dynamics of a system moving

382 though IPSs (Figure 2A). 
Low Dictionary

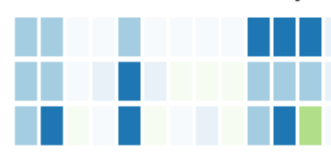

High Dictionary
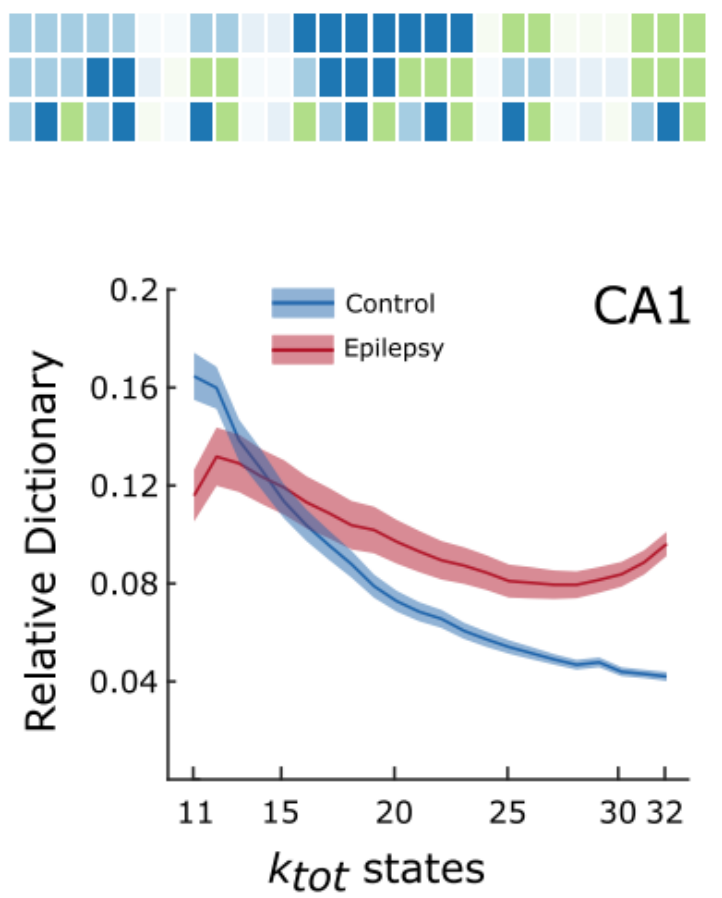

B

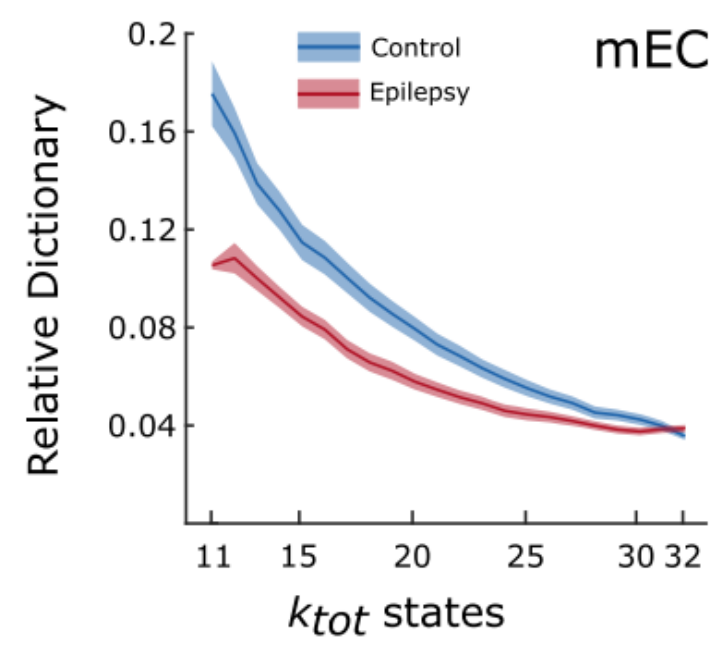

Figure 5 - Relative dictionaries within the libraries - (A) Fictional cartoons representing two

385 extremes for the measure of relative dictionary. Each row represents a feature (firing, storage,

386 sharing); for simplicity we do not take into account the brain states (THE and SO). We consider

387 three substates (light blue, dark blue, green) per feature (using the same color code for

388 simplicity), which makes a total of $3^{3}=27$ words (the representation is similar to counting in

389 base 3 with color, increasing from left to right). Words that are not observed are shaded. A low

390 relative dictionary (left) contains a low fraction of all possible words, while a high relative

391 dictionary (right) contains a high fraction. (B) Relative dictionary values as a function of $k_{\text {tot }}$. As

392 expected, the fraction of words used in control decreases as the number of possible words

393 increases. The relative dictionaries are similar in mEC and CA1 in controls. There is a marked

394 decrease in the relative dictionary in the $\mathrm{mEC}$ in epilepsy. In CA1, the relative dictionary in

395 increased or decreased as compared to control as a function of $k_{\text {tot }}$. Blue is representative of 
control data and red is representative of epileptic data. The bold lines are the mean, and the

397 shaded regions are the $99 \%$ bootstrapped confidence interval.

399 The syntax of substate sequences is less regular in epilepsy

400 Compressibility is a key property of an object as it represents the degree of internal order of the

401 object. This is because any regularity within may be described by simply referencing its previous

402 occurrence. Again, our state tables are bordered by two extreme cases: order and randomness

403 (Figure 6A). An ordered table is dominated by a highly structured syntax, typically dominated by

404 a lower dictionary and long periods of sustained words. Therefore, an ordered table is very

405 compressible due to this internal order. A random table, on the other hand, typically contains an

406 exceedingly high number of words, which follow each other in a disorderly (random) manner.

407 This results in non-compressibility. A complex table is one that lies between those extremes. In

408 order to characterize the complexity of the state tables, we compute a tailored form of

409 description length complexity (Clawson et al., 2019; Rissanen, 1978), which is scaled to the sister

410 libraries of order and disorder. Thus, in Figure 6B, 0 represents the complexity of the ordered

411 library, something very compressible, while 1 represents the complexity of our disordered library,

412 something very uncompressible (as shown in Figure 6A). In controls, the complexity is similar in

413 mEC and CA1, close to an ordered table. In epilepsy, the complexity is significantly increased for

414 all $k_{\text {tot }}$ values, while it is increased in the $\mathrm{mEC}$ at the high end of our library.

416 Combining the results from Figures 5 \& 6, we can propose the following interpretation. In CA1, 417 the increase in complexity found in epilepsy, at least for books with sufficiently large $k_{\text {tot }}$, can be 
418 explained, at least in part, by an enriched dictionary, since enrichment of the relative dictionary

419 positively correlate with complexity (Clawson et al., 2019). In the mEC, the relative dictionary

420 decreases while the complexity mildly increases. Thus, mEC books have a less regular syntax

421 despite being constructed out of a lesser number of words.

A

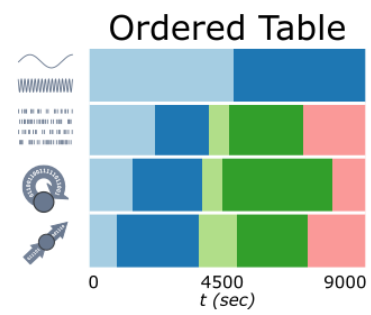

B

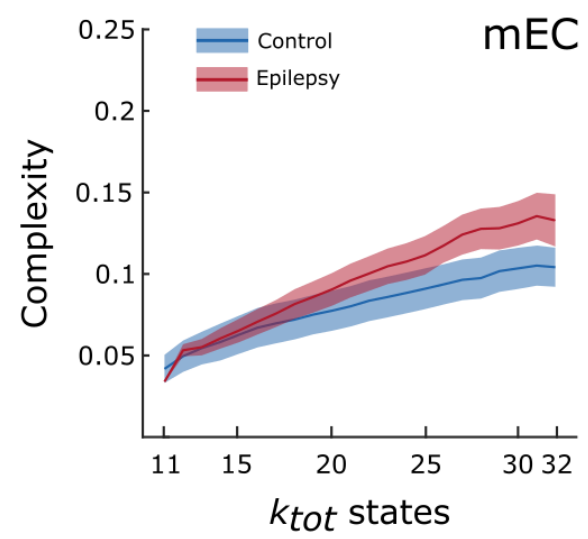

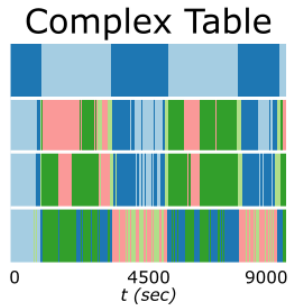

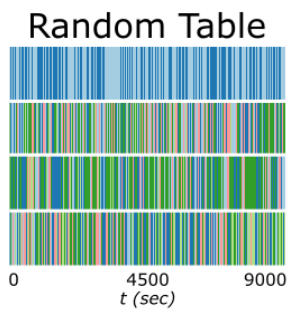

423 Figure 6 - Order, disorder, and complexity - (A) Examples of state tables, similar to that of Fig

$4242 \mathrm{~A}$, from the mEC showing the two extremes of order and disorder as well as one of the possible

425 state tables taken from the state table library. (B) Complexity values for both the mEC and CA1

426 as a function of $k_{\text {tot }}$. The complexity is similar in mEC and CA1 in controls. In epilepsy, the

427 complexity is largely increased in CA1, and only for large $k_{\text {tot }}$ values for mEC. Blue is representative

428 of control data and red is representative of epileptic data. The bold lines are the mean, and the

429 shaded regions are the $99 \%$ bootstrapped confidence interval. 


\section{Discussion}

431 This study provides evidence that epileptic conditions alter information processing in its simplest

432 sense, the primitive storage and sharing operations as we introduce here, in a brain-region

433 dependent manner. As these basic processes are necessarily involved in a variety of neural

434 computations, their alterations may indirectly impact numerous cognitive functions.

436 The main limitation to our study is that it is made under anesthesia, versus for example, goal-

437 directed behavior to assess cognitive function. The type of analysis we performed is powerful as

438 it allows unraveling basic properties of information processing without needing to know which

439 computations are ongoing. However, it requires long-duration, stable recordings with large state

440 sampling to obtain enough data points to perform reliable statistics. We did not record during

441 natural sleep, because seizures and interictal spikes (which would act as strong confounding

442 factors) mostly occur during the light phase, while they do not occur under anesthesia. However,

443 a similar type of analysis performed in control animals led to similar results during sleep and

444 anesthesia (Clawson et al., 2019), suggesting that the anesthesia procedure we use does not

445 significantly alter core information dynamics.

447 We refer to the elementary information storage and sharing operations as primitive (or low level)

448 information processing operations, as we consider them as fundamental building blocks within

449 an algorithm to reach an end condition (like a function), similar to the "algorithmic level",

450 introduced by Marr \& Poggio (1977). Algorithm is used here in its most generic meaning, as we

451 do not claim that the brain is analogous to a computer. Such primitive processing operations, as 
452 we define them, represent nothing else than the emergent "informational effect" of very

453 concrete neurophysiological phenomena. Storage and sharing of information directly derive from

454 auto- and cross-correlations in firing, which widely vary in neuronal populations (Schneidman et

455 al., 2006), and can be directly measured from spiking activity of neurons. Other primitive

456 processing operations exist, such as information transfer (Palmigiano et al., 2017; Schreiber,

457 2000) or information modification (Lizier et al., 2013; Wibral et al., 2017). Our recordings and

458 choice of a time-resolved approach do not provide enough data to track these more sophisticated

459 operations. However, the processing functions of storage and sharing are especially important as

460 they represent statistical measures of information flow in time, and spacetime, respectively.

462 We show that primitive information processes are organized in temporal sequences of

463 information processing substates (IPSs), which are extracted via a cluster analysis. We have used

464 a non-biased approach, spanning many possible combinations of numbers of clusters. The fact

465 that most results are independent from the choice of the number of clusters provides a strong

466 argument for the genericity of our conclusions. With this approach, we demonstrate a

467 degradation of complexity due to enhanced randomness in epilepsy. This conclusion stems from

468 the convergence of complementary analyses. First, storage and sharing hubs are less robust,

469 waxing and waning in a more erratic manner across substates and the recruitment of neurons

470 into the integrated core of sharing networks is more volatile. Second, average storage and

471 sharing strength are more similar between brain states, and this "dedifferentiation" occurs

472 despite the higher contrast between substates. Third, the state specificity of IPS is reduced, i.e.,

473 many IPSs are now redundant between THE or SO. Together, these results imply that a change in 
474 brain state is no longer associated to strong specificity in information processing. Fourth, freed

475 from the constraint of being strongly state-specific, the relative dictionary in epilepsy could, in

476 principle, be increased. However, mEC has a decreased relative dictionary, which instead implies

477 an ability to form unique IPSs. Yet, the description complexity of IPS sequences tends to be larger

478 in epilepsy than control. In other words, IPS sequences have a less regular syntax despite being

479 assembled out of less unique words.

481 The IPS dynamics of CA1 show, in general, less alterations than that of mEC. The fact that 482 information processing is affected in brain region-dependent manner is an important result. The 483 mEC and CA1 have distinct cytoarchitectures and different fates following an epileptogenic insult.

484 Most striking is the loss of layer 3 in the mEC, and the injury of many pyramidal cells and 485 interneurons in the CA1 region (Curia et al., 2008). It is not possible to assign a given alteration 486 in information processing to a given morpho-functional changes in the mEC or CA1. Global brains 487 states (THE and SO) and IPSs are emergent properties. Any change in any brain region can 488 potentially affect neuronal dynamics anywhere from the local to the global scale. Therefore, the 489 morpho-functional alterations in MEC or CA1 may contribute to any combination of local and 490 global changes. However, changes in terms of information processing do not necessarily have to 491 be homogenous across brain regions. In fact, brain region-specific modifications are expected as 492 each region is embedded in different functional networks. How these brain-region specific 493 changes contribute to comorbidities (such as cognitive deficit, anxiety, and depression) remain 494 to be determined. 
Our measure of complexity is that of compressibility, accounting for the internal structure, i.e.,

497 how internally ordered are IPS syntaxes. Any change in this internal organization would thus

498 imply an underlying change in algorithmic operation, resulting in different computation in control

499 and epilepsy conditions. Our measure of complexity does not allow distinguishing beween an

500 increase in processing versus an increase in noise, as complexity would grow in both cases. Other

501 measures can be used, but they would require more data (Crutchfield, 2011). However, in CA1,

502 books with large $k_{\text {tot }}$ have an increased, rather than decreased dictionary size, which may explain

503 the strong increase in sequence complexity. It is not clear, however, that this dictionary increase

504 is a positive factor as it may reflect a more irregular IPS selection, with rare IPSs indicating

505 dysfunction in IPS sequential production. Another possibility is that boosted IPS sequence

506 complexity in CA1 and, at a lesser extent, $\mathrm{mEC}$ is a compensatory mechanism to generate a more

507 sophisticated syntax to compensate for other shortages, such as reduced hub stability and

508 degraded state-specificity of IPS.

510 In a biological context, the algorithmic level change comes as a result of altered collective, spiking

511 activity and could lead to an entirely different expression of higher-level behavior, such as

512 cognition. However, the question of whether this increase of complexity (decrease of internal

513 order) observed in epilepsy is the source of cognitive deficits or not remains ultimately open. It

514 has been theorized that "biological systems manipulate spatial and temporal structure to

515 produce order - low variance - at local scales" in an effort to adapt and survive (Flack, 2019).

516 Therefore, if networks are still functional in epilepsy conditions, are these manipulations now

517 less effective? Or is the resulting low variance order now too difficult to sustain due to a 
518 combination of physiological and functional changes? These issues remain to be addressed.

519 Nevertheless, the approaches presented here introduce valuable insight into aspects of the

520 collective behavior of neural populations, and provide a quantitative framework to answer such

521 questions.

523 In conclusion, the framework we introduce here to compare information processing between

524 control and epilepsy, can be generalized to neurological disorders. Since most, if not all, of the

525 latter, including migraine, Alzheimer's disease, and Parkinson's disease are associated with co-

526 morbidities, it will be particularly interesting to determine whether information processing at the

527 algorithmic level is also affected in these disorders. Following the principle of degeneracy (Prinz

528 et al., 2004), very different structural alterations, which characterize different neurological

529 disorders, may produce similar alterations in information processing, providing an explanation

530 for the commonalities of co-morbidities across different disorders.

532 Methods

533 Ethics

534 All experiments were conducted in accordance with Aix-Marseille Université and Inserm

535 Institutional Animal Care and Use Committee guidelines. The protocol was approved by the

536 French Ministry of National Education, Superior Teaching, and Research, under the authorization

537 number 01451-02. All surgical procedures were performed under anesthesia and every effort

538 was made to minimize suffering and maximize the animals' wellbeing from their arrival to their

539 death. All the animals were housed in pairs in large cages with minimal enrichment, food and 
540 water at libitum, in a room with controlled environment (temperature: $22 \pm 1{ }^{\circ} \mathrm{C} ; 12 \mathrm{~h}$ light/dark

541 schedule with lights off at 8:00 pm; hygrometry: 55\%; ventilation: $15-20 \mathrm{vol} / \mathrm{h}$ ).

\section{Data information.}

543 We use in this work a portion of the data (5 out of 7 original experiments) initially published by

544 Clawson et al. 2019 as control data, which includes local field potentials (LFPs) and single-unit

545 recordings obtained from the dorsomedial entorhinal cortex ( $\mathrm{mEC}$ ) and the dorsal hippocampus

546 (HPC) of anesthetized rats. Six recordings are original data, which includes LFPs and single-units

547 recorded in the $\mathrm{mEC}$ and HPC recorded simultaneously under anesthesia in epileptic condition.

548 See Figures S1 for details on recordings, number of cells, and layers recorded.

549 Epilepsy model and surgery.

550 We induced status epilepticus (SE) on 6 male Wistar (250-400 g; Charles Rivers) by a single

551 intraperitoneal (IP) injection of pilocarpine (320 mg/kg; Sigma-Aldrich), one week after receiving

552 the animals from the vendor. To reduce peripheral effects, rats were pre-treated with methyl-

553 scopolamine (1 mg/kg, IP; Sigma-Aldrich) 30 min before the pilocarpine injection. SE was stopped

554 by diazepam (10 mg/kg, IP, two doses within a 15 -min interval) after $60 \mathrm{~min}$. Then the animals

555 were hydrated with saline $(2 \mathrm{ml}, \mathrm{IP}$, twice within $2 \mathrm{~h}$ ) and fed with a porridge made of soaked

556 pellets, until they resumed normal feeding behavior.

557 At least 8 weeks after SE induction, we performed acute recordings. Rats were first quickly placed

558 in isoflurane $\left(4 \%\right.$ in $\left.21 / \mathrm{min}_{2}\right)$ and injected IP with urethane $(1.5 \mathrm{~g} / \mathrm{kg})$ and ketamine/xylazine $(20$

559 and $2 \mathrm{mg} / \mathrm{kg}, \mathrm{IM}$ ), additional doses of ketamine/xylazine ( 2 and $0.2 \mathrm{mg} / \mathrm{kg}$ ) being supplemented

560 during the electrophysiological recordings. At all times the body temperature was monitored and

561 kept constant with a heating pad. Heart rate, breathing rate, pulse distension, and arterial oxygen 
562 saturation were also monitored with an oximeter (MouseOX; StarrLife Sciences) during the

563 duration of the experiment to ensure the stability of the anesthesia and monitor the vital

564 constants. The head was fixed in a stereotaxic frame (Kopf) and the skull was exposed and

565 cleaned. Two miniature stainless-steel screws driven into the skull above the cerebellum served

566 as ground and reference electrodes. Two craniotomies were performed to reach the $\mathrm{mEC}$ and

567 the CA1 field of the HPC, respectively: from bregma: $-7.0 \mathrm{~mm} \mathrm{AP}$ and $+4.0 \mathrm{~mm} \mathrm{ML}$; and from

568 bregma: $-3.0 \mathrm{~mm} \mathrm{AP}$ and $+2.5 \mathrm{~mm} \mathrm{ML}$. We chose these coordinates to respect known anatomical

569 and functional connectivity in the cortico-hippocampal circuitry (Witter et al., 1988; Witter et al.,

570 1989). Two 32-site silicon probes (NeuroNexus) were mounted on a stereotaxic arm each. A

$571 \mathrm{H} 1 \times 32-10 \mathrm{~mm}-50-177$ was lowered at 5.0-5.2 $\mathrm{mm}$ from the brain surface with a $20^{\circ}$ angle to reach

572 the dorso-medial portion of the mEC, and a H4x8-5mm-50-200-177 probe was lowered at 2.5

$573 \mathrm{~mm}$ from the brain surface with a $20^{\circ}$ angle to reach dorsal CA1. The on-line positioning of the

574 probes was assisted by: the presence of unit activity in cell body layers and the reversal of theta

575 ([3 6] Hz in anesthesia) oscillations when passing from layer 2 to 1 for the mEC probe, and the

576 presence in stratum pyramidale either of unit activity and ripples $(80-150 \mathrm{~Hz})$ for the HPC probe.

577 At the end of the recording, the animals were injected with a lethal dose of Pentobarbital Na

578 (150mk/kg, i.p.) and perfused intracardially with 4\% paraformaldehyde solution. We confirmed

579 the position of the electrodes (DilC18(3) (catalog \#46804A, InterChim) was applied on the back

580 of the probe before insertion) histologically on $40 \mu \mathrm{m}$ Nissl-stained section as reported previously

581 in detail (Ferraris et al., 2018; Quilichini et al., 2010). We used only experiments with appropriate

582 position of the probe for analysis.

583 Data collection and spike sorting. 
584 Extracellular signal recorded from the silicon probes was amplified (1000x), bandpass filtered (1

$585 \mathrm{~Hz}$ to $5 \mathrm{kHz}$ ) and acquired continuously at $32 \mathrm{kHz}$ with a 64-channel DigitalLynx (NeuraLynx) at

586 16-bit resolution. We preprocessed the raw data using a custom-developed suite of programs

587 (Csicsvari et al., 1999). The signals were down-sampled to $1250 \mathrm{~Hz}$ for the local field potential

588 (LFP) analysis. Spike sorting was performed automatically, using KLUSTAKWIK

589 (http://klustakwik.sourceforge.net (Harris et al., 2000)), followed by manual adjustment of the

590 clusters, with the help of auto-correlogram, cross-correlogram and spike waveform similarity

591 matrix (KLUSTERS software package, http://klusters.source-forge.net (Hazan et al., 2006)). After

592 spike sorting, we plotted the spike features of units as a function of time, and we discarded the

593 units with signs of significant drift over the period of recording. Moreover, only units with clear

594 refractory periods and well-defined cluster were included in the analyses (Harris et al., 2000).

595 Recording sessions were divided into brain states of theta (THE) and slow oscillation (SO) periods

596 using a visual selection from the ratios of the whitened power in the HPC LFP [3 6] Hz theta band

597 and the power of the mEC LFP neighboring bands ([1 3] $\mathrm{Hz}$ and [7 14] Hz), and assisted by visual

598 inspection of the raw traces (Ferraris et al., 2018; Quilichini et al., 2010). We then used band-

599 averaged powers over the same frequency ranges of interest as features for the automated

600 extraction of spectral states via unsupervised clustering, which confirmed our manual

601 classification. We determined the layer assignment of the neurons from the approximate location

602 of their soma relative to the recording sites (with the largest- amplitude unit corresponding to

603 the putative location of the soma), the known distances between the recording sites, and the

604 histological reconstruction of the recording electrode tracks. Animals were recorded for at least

605 two hours in order to get few alternations of THE and SO episodes. 


\section{Feature Computation}

607 As in our previous work, for each region recorded we computed 4 main features from the 608 electrophysiological data: global oscillatory band, neuronal firing sets, active information storage 609 and the information sharing. We also keep the same sliding window paradigm where each

610 feature is computed within a 10 second window, and then the window is then moved forward in

611 time 1 second, which gives a 9 second overlap. Therefore, when features are computed as

612 described below, they are computed in this windowed fashion. The global oscillatory band

613 features were computed by examining the LFP from both EC and CA1 and computing spectral

614 power within 8 unequally sized frequency ranges $(0-1.5 \mathrm{~Hz}, 1.5-2 \mathrm{~Hz}, 2-3 \mathrm{~Hz}, 3-5 \mathrm{~Hz}, 5-7 \mathrm{~Hz}, 7-$

$61510 \mathrm{~Hz}, 10-23 \mathrm{~Hz}$ and $23-50 \mathrm{~Hz}$ ), averaged over all channels within each of the recorded layers.

616 Firing sets, active information storage, and the information sharing networks were all computed

617 using a binarized raster built from the temporal labeling of spike firing (see Data Collection and

618 Spike Sorting). Spiking data was binned using a $50 \mathrm{~ms}$ bin; if a neuron fired within a given bin the

619 output is a ' 1 ', and if not, a ' 0 '. This, for example would mean that a 2 -hour recording would be

620 transformed from a 7200 second $\times N$ neuron matrix to a $7200000 \times N$ neuron matrix that is

621 composed solely of 0 's and 1's. Firing sets were computed by computing the average firing

622 density for each neuron within a window, and after these averages were compiled into time-

623 dependent vectors. This resulting matrix is the Firing Features. Active information storage was

624 computed by measuring the mutual information of a neuron's binarized spike train between a

625 given window and the window previous. What active information storage seeks to capture is the

626 temporal ordering of individual spiking neurons, rather than capturing neurons that fire

627 temporally close to one another (such as in the firing features). The resulting matrix is the Storage 
628 Features. Information sharing is computed by measuring the mutual information between a given

629 neuron's binarized spike train within a window and another neuron's binarized spike train in the

630 window previous. This process is iterated over all possible neuron pairs. Information sharing

631 captures a similar metric to that of active information storage, although the key difference is that

632 information sharing captures not just the temporal ordering, but the spatio-temporal ordering of

633 spike timing, as it is computed across neuron pairs, rather than individual neurons. The resulting

634 matrix is the Information Sharing. Although these measures have only been briefly described

635 here, we suggest to the interested reader to examine the methods presented in our previous

636 work [REF] where they have been rigorously defined.

\section{Feature-Based Substate Extraction}

639 State extraction for each recording were also computed using the methods of our previous work,

640 namely based around k-means clustering of each feature. The exception here, is we no longer

641 choose a stable number of $\mathrm{K}$ clusters in k-means. Rather we cluster our 3 raster-based computed

642 features (firing, storage, sharing) 3 separate times with $K$ ranging from $K=3,4, \ldots 10$. The function

643 'kmeans' was used from the default MATLAB toolbox. More information can be found on the

644 Mathworks website. These $\mathrm{K}$ values were chosen as they represented a clustering range of too

645 gross to too fine based on previous findings. $K<=2$ would represent the same, or less, number

646 of states as global states, which was previously established to be too small (Clawson et al., 2019).

647 The clustering became too fine when $K>=10$, wherein many substates only appeared for brief

648 time periods, and never re-occurred. For each feature there are 8 different clustering results,

649 done in an unsupervised manner 3 times to ensure that our results do not rely on single instance 
650 of clustering. This gave our analysis an opportunity to compute all metrics defined below over a

651 robust range of $\mathrm{K}$, ensuring that we can investigate how our substate stable metrics and results

652 vary with arbitrarily too little or too many substates.

653

654 To compute the null model for substate extraction the process detailed above was repeated with

655 the time stamps of all firing, storage and sharing jittered. This therefore retains the global mean

656 and variance. Then, k-means was run on this jittered dataset 3 times, to produce 3 different

657 clustering of the randomized dataset. These were not modified after this step and were used in

658 any instances where a null model was needed (i.e. for silhouette and contrast).

660 Substate Tables

661 Our main meta-object of study is a state table, a combination of our four features into a matrix

662 ( 4 x number of windows). Table generation is an iterative process, as we have 8 possible substate

663 configurations per feature. First, $k=3$ in cluster attempt 1 for firing (FIRE $\left.k 3 C_{1}\right), k=3$ in cluster

664 attempt 1 for storage (STORE $\left.\mathrm{K}_{\mathrm{K} 31}\right)$, and $\mathrm{k}=3$ in cluster attempt 1 for sharing (SHARE $\mathrm{K}_{\mathrm{K} 31}$ ), are used

665 in conjunction with the clustered spectral substates to form substate table 1 (Figure 2A).

667 Then, FIRE ${ }_{K 3 C 1}, \mathrm{STORE}_{\mathrm{K} 3 \mathrm{C1}}$, and $\mathrm{SHARE}_{\mathrm{K} 4 \mathrm{C1}}$ are used in conjunction with the clustered spectral

668 substates to form substate table 2. After, FIRE ${ }_{\mathrm{K} 3 \mathrm{C} 1}, \mathrm{STORE}_{\mathrm{K} 3 \mathrm{C1}}$, and $\mathrm{SHARE}_{\mathrm{K} 5 \mathrm{C} 1}$ used in conjunction

669 with the clustered spectral substates to form substate table 3 . This process continues such that

670 all combinations of possible $\mathrm{k}$ values have been saved for a total of 512 different substate tables,

671 with the final table having FIRE ${ }_{K 10 C 1}, S_{\text {STORE }} 10 C 1$, and SHARE $E_{K 10 C 1}$. It is important to note that all 
672 tables have the same spectral clustering, as the 2 substates of SO and THE are extremely robust

673 as discussed above. This entire process is then repeated for each clustering attempt, resulting in

6743 sets of our 512 substate tables for each region for each recording. Where applicable, all results

675 are given as a function of total k states per table (i.e. for state table 1 , there are 2 global states,

6763 firing, 3 storage and 3 sharing for a total $\left.k_{\text {total }}=11\right)$.

678 To produce the ordered tables for the 'ordered' null model, each substate table was sorted such

679 that all substates with label ' 1 ' appeared first, label ' 2 ' was second, and so on and so forth. This

680 can easily be achieved with the MATLAB function sort. Note that there is only one possible

681 version of this type of ordering, and therefore the sample size for ordered tables is the same as

682 recordings ( $n=5$ for control, $n=6$ for epilepsy). To produce the randomized tables, substate

683 labels were randomly permuted in time. For this process, we used bootstrapping to produce as

6845000 randomizations to ensure the random null model was as strong as possible. To do this, $90 \%$

685 of each table was taken, randomly permuted and saved. These resulting tables were used as the

686 random null model for relative dictionary and complexity seen in Figure 5 \& 6 .

\section{Contrast}

689 To calculate contrast for a given feature we first calculate its global mean for each neuron (i.e., 690 global mean firing per neuron). Here, 'global' refers to the entire recording. We then calculate 691 the substate mean for each neuron by concatenating all periods of a given substate and 692 calculating the mean across the 'entire' substate. The formula for contrast is then defined as the 
693 difference between the substate mean firing rate and the global mean firing rate, normalized by

694 the global mean firing rate.

$$
\text { contrast }=\frac{\mu_{\text {substate }}-\mu_{\text {global }}}{\mu_{\text {global }}}
$$

696 This allows the contrast to be either positive or negative. This process was done for all 3 features

697 of firing, storage and sharing such that there are contrast values for each. This process was

698 repeated for all possible clustering, therefore a contrast value per feature per $k$.

\section{Substate-Specificity}

701 To compute the distribution of substates within periods of SO and THE we counted the number

702 of times a substate appeared within a given epoch. Some substates exclusively appeared in only

703 SO or THE, while others occurred in both. From these frequencies we estimated $p(T H E)$ and

$704 \mathrm{p}(\mathrm{SO})$, i.e. the probability of a given substate occurring in either THE or SO, respectively. SSI is

705 then:

$$
S S I=|p(T H E)-p(S O)|
$$

707 This equation results in SSI bound between 0 and 1, where 1 represents a state who exclusively occurs in either THE or SO and 0 represents a state that occurs equally in THE and SO.

711 In this work we define a hub neuron in the same way as our previous work. Namely, for a given

712 feature if a neuron's activity within a given substate was higher than the $90^{\text {th }}$ percentile it was

713 marked as a hub for the feature for that state. We compute hubs for every iteration of state table

714 as defined above, such that we have a graph, or matrix, (see FIG 4A) for each state table. These 
715 matrices are Neuron $\times k_{\text {total }}$ where each entry is either a ' 0 ' for non-hub or ' 1 ' for hub. To compute

716 how stable each of these matrices are as a function of $k$, we compute the normalized hamming

717 distance of each matrix using the pdist2 function in MATLAB but modified so that it gives a sense

718 of how stable hubs are across states, where perfect similarity would result in a ' 1 ', and no

719 similarity at all would give a '0'.

\section{Coreness \& Jaccard}

722 The values for coreness \& Jaccard were computed using the methods presented in Pedreschi et

723 al. (2020). These were then analyzed using the same sliding window technique as presented in

724 'Feature Computation'. After, periods of THE and SO were analyzed with similar techniques as 725 that of Figure 1.

\section{Dictionary \& Complexity}

728 To compare sequences of substates of different types or in different regions we introduced a

729 symbolic description of substate switching. With this description, each substate label acts as a

730 letter symbol $s^{(p)}$, where $(p)$ can indicate firing, sharing or storage. For example, the firing features

731 from the example substate table 1 [FIG 2A] would have the integer labels 1, 2, 3, and 4 (they can

732 also arbitrarily be assigned letters as well, i.e. A, B, and C). We can therefore describe the

733 temporal sequences of the visited substates of each feature as an ordered list of integers $s^{(p)}(t)$.

734 Once substate labels are thought of as letters, we define the combination of firing, storage and

735 sharing letters in each state table from a given window as 3 letter words. Using the formalism of

736 linguistics, we can then compute the dictionary, or the number of words expressed, of a given 
737 recording within a region. We can also compute the used dictionary fraction, or the number of

738 words found in the dictionary divided by the number of theoretically possible words given the

739 number of substates per feature. For example, substate table 1 could have expressed 27 unique

740 words. The used dictionary fraction was computed in an identical way to that of Clawson et al

7412019. Specially, see 'Complexity of substates sequences.

743 Using these methods, we compute the complexity of the sequences expressed using the notions

744 of Kolmogorov-Chaitin complexity and minimum description length approaches (Crutchfield,

745 2011). While further discussion of method can be found here (Clawson et al., 2019) - the aspects

746 of this complexity measure that is relevant for this work is that a random sequence of letters (and

747 words) produces a higher complexity, while an ordered sequence of letters (and words) would

748 produce a low complexity.

750 Ordered \& Random Substate Tables

751 To have relevant points of reference in our measures, each substate table was ordered and

752 randomized. For the case of ordering, all substate labels for all features were sorted in ascending

753 order which keeps the total lifetime of any state constant, while removing the temporal

754 organization in an ordered fashion. In the case of randomization, all substate labels for all features

755 were randomized 500 times, which again keeps the total lifetime of any state constant, while

756 removing the temporal organization in a random fashion.

757 To compute the relative minimums and maximums for comparisons between order and random

758 the MATLAB function 'rescale' was used. The minimums were computed using the average (of a 
given measure) of all ordered state tables for a given $k_{\text {total }}$ and the maximums were computed

using the average (of a given measure) of all random substate tables for a given $k_{\text {total. }}$

Plotting

Various tools were used for plotting. While mostly done via MATLAB, other tools were also

used from 'Moving Beyond p-values' (Ho et al., 2019).

Bibliography

Barba, C., Barbati, G., Minotti, L., Hoffmann, D., \& Kahane, P. (2007, Jul). Ictal clinical and scalp-EEG findings differentiating temporal lobe epilepsies from temporal 'plus' epilepsies. Brain, 130(Pt 7), 1957-1967. https://doi.org/10.1093/brain/awm108

Bartolomei, F., Chauvel, P., \& Wendling, F. (2008, Jul). Epileptogenicity of brain structures in human temporal lobe epilepsy: a quantified study from intracerebral EEG. Brain, 131(Pt 7), 1818-1830. https://doi.org/10.1093/brain/awn111

Blumcke, I., Thom, M., Aronica, E., Armstrong, D. D., Bartolomei, F., Bernasconi, A., Bernasconi, N., Bien, C. G., Cendes, F., Coras, R., Cross, J. H., Jacques, T. S., Kahane, P., Mathern, G. W., Miyata, H., Moshe, S. L., Oz, B., Ozkara, C., Perucca, E., Sisodiya, S., Wiebe, S., \& Spreafico, R. (2013, Jul). International consensus classification of hippocampal sclerosis in temporal lobe epilepsy: a Task Force report from the ILAE Commission on Diagnostic Methods. Epilepsia, 54(7), 1315-1329. https://doi.org/10.1111/epi.12220

Calhoun, V. D., Miller, R., Pearlson, G., \& Adali, T. (2014, Oct 22). The chronnectome: time-varying connectivity networks as the next frontier in fMRI data discovery. Neuron, 84(2), 262-274. https://doi.org/10.1016/j.neuron.2014.10.015

Chauvière, L., Rafrafi, N., Thinus-Blanc, C., Bartolomei, F., Esclapez, M., \& Bernard, C. (2009). Early deficits in spatial memory and theta rhythm in experimental temporal lobe epilepsy. Journal of Neuroscience, 29(17), 5402-5410. https://doi.org/29/17/5402 [pii] 10.1523/JNEUROSCI.469908.2009

Clawson, W., Vicente, A. F., Ferraris, M., Bernard, C., Battaglia, D., \& Quilichini, P. P. (2019, Jun). Computing hubs in the hippocampus and cortex. Sci Adv, 5(6), eaax4843. https://doi.org/10.1126/sciadv.aax4843 
Crutchfield, J. P. (2011). Between order and chaos. Nature Physics, 8(1), 17-24. https://doi.org/10.1038/nphys2190

796

797

798

799

800

801

802

803

804

805

806

807

808

809

810

811

812

813

814

815

816

817

818

819

820

821

822

823

824

825

826

827

828

829

830

831

832

833

Csicsvari, J., Hirase, H., Czurko, A., Mamiya, A., \& Buzsáki, G. (1999). Oscillatory coupling of hippocampal pyramidal cells and interneurons in the behaving Rat. Journal of Neuroscience, 19(1), 274-287. https://doi.org/10.1523/JNEUROSCl.19-01-00274.1999

Curia, G., Longo, D., Biagini, G., Jones, R. S., \& Avoli, M. (2008, Jul 30). The pilocarpine model of temporal lobe epilepsy. J Neurosci Methods, 172(2), 143-157. https://doi.org/10.1016/i.jneumeth.2008.04.019

de Barros Lourenco, F. H., Marques, L. H. N., \& de Araujo Filho, G. M. (2020, Jul). Electroencephalogram alterations associated with psychiatric disorders in temporal lobe epilepsy with mesial sclerosis: A systematic review. Epilepsy Behav, 108, 107100. https://doi.org/10.1016/i.yebeh.2020.107100

Ferraris, M., Ghestem, A., Vicente, A. F., Nallet-Khosrofian, L., Bernard, C., \& Quilichini, P. P. (2018, Mar 21). The Nucleus Reuniens Controls Long-Range Hippocampo-Prefrontal Gamma Synchronization during Slow Oscillations. J Neurosci, 38(12), 3026-3038. https://doi.org/10.1523/JNEUROSCI.3058-17.2018

Flack, J. C. (2019). Life's Information Hierachy. Sante Fe Institute Press.

Harris, K. D., Henze, D. A., Csicsvari, J., Hirase, H., \& Buzsáki, G. (2000). Accuracy of tetrode spike separation as determined by simultaneous intracellular and extracellular measurements. Journal of Neurophysiology, 84(1), 401-414.

Hazan, L., Zugaro, M., \& Buzsáki, G. (2006). Klusters, NeuroScope, NDManager: a free software suite for neurophysiological data processing and visualization. J Neurosci Methods, 155(2), 207-216. https://doi.org/10.1016/i.jneumeth.2006.01.017

Hesdorffer, D. C. (2016, Jun). Comorbidity between neurological illness and psychiatric disorders. CNS Spectr, 21(3), 230-238. https://doi.org/10.1017/S1092852915000929

Ho, J., Tumkaya, T., Aryal, S., Choi, H., \& Claridge-Chang, A. (2019, Jul). Moving beyond P values: data analysis with estimation graphics. Nat Methods, 16(7), 565-566. https://doi.org/10.1038/s41592-019-0470-3

Holmes, G. L. (2015, Jun). Cognitive impairment in epilepsy: the role of network abnormalities. Epileptic Disord, 17(2), 101-116. https://doi.org/10.1684/epd.2015.0739 
834

835

836

837

838

839

840

841

842

843

844

845

846

847

848

849

850

851

852

853

854

855

856

857

858

859

860

861

862

863

864

865

866

867

868

869

870

871

872

873

874

Inostroza, M., Brotons-Mas, J. R., Laurent, F., Cid, E., \& de la Prida, L. M. (2013, Nov 6). Specific impairment of "what-where-when" episodic-like memory in experimental models of temporal lobe epilepsy. J Neurosci, 33(45), 17749-17762. https://doi.org/10.1523/JNEUROSCI.0957$\underline{13.2013}$

Kirst, C., Timme, M., \& Battaglia, D. (2016, Apr 12). Dynamic information routing in complex networks. Nat Commun, 7(7), 11061. https://doi.org/10.1038/ncomms11061

Krishnan, V. (2020, Jul 14). Depression and Anxiety in the Epilepsies: from Bench to Bedside. Curr Neurol Neurosci Rep, 20(9), 41. https://doi.org/10.1007/s11910-020-01065-z

Lenck-Santini, P. P., \& Holmes, G. L. (2008, May 7). Altered phase precession and compression of temporal sequences by place cells in epileptic rats. J Neurosci, 28(19), 5053-5062. https://doi.org/10.1523/JNEUROSCI.5024-07.2008

Lenck-Santini, P. P., \& Scott, R. C. (2015, Sep 3). Mechanisms Responsible for Cognitive Impairment in Epilepsy. Cold Spring Harb Perspect Med, 5(10). https://doi.org/10.1101/cshperspect.a022772

Lizier, J. T., Atay, F. M., \& Jost, J. (2012, Aug). Information storage, loop motifs, and clustered structure in complex networks. Phys Rev E Stat Nonlin Soft Matter Phys, 86(2 Pt 2), 026110. https://doi.org/10.1103/PhysRevE.86.026110

Lizier, J. T., Flecker, B., \& Williams, P. L. (2013). Towards a Synergy-based Approach to Measuring Information Modification. 2013 leee Symposium on Artificial Life (Alife), 43-51. https://doi.org/10.1109/ALIFE.2013.6602430

Lopez-Pigozzi, D., Laurent, F., Brotons-Mas, J. R., Valderrama, M., Valero, M., Fernandez-Lamo, I., Cid, E., Gomez-Dominguez, D., Gal, B., \& Menendez de la Prida, L. (2016, Nov-Dec). Altered Oscillatory Dynamics of CA1 Parvalbumin Basket Cells during Theta-Gamma Rhythmopathies of Temporal Lobe Epilepsy. eNeuro, 3(6). https://doi.org/10.1523/ENEURO.0284-16.2016

Marr, D. C., \& Poggio, T. (1977). From Understanding Computation to Understanding Neural Circuitry. Neurosciences Research Program Bulletin, 15(3), 470-491. <Go to ISI>://WOS:A1977EH37300024

Palmigiano, A., Geisel, T., Wolf, F., \& Battaglia, D. (2017, Jul). Flexible information routing by transient synchrony. Nat Neurosci, 20(7), 1014-1022. https://doi.org/10.1038/nn.4569

Pedreschi, N., Bernard, C., Clawson, W., Quilichini, P., Barrat, A., \& Battaglia, D. (2020). Dynamic coreperiphery structure of information sharing networks in entorhinal cortex and hippocampus. Network Neuroscience, 1-30. https://doi.org/10.1162/netn a 00142 
912

913

914

Porta, A., Baumert, M., Cysarz, D., \& Wessel, N. (2015, Feb 13). Enhancing dynamical signatures of complex systems through symbolic computation. Philos Trans A Math Phys Eng Sci, 373(2034). https://doi.org/10.1098/rsta.2014.0099

Prinz, A. A., Bucher, D., \& Marder, E. (2004, Dec). Similar network activity from disparate circuit parameters. Nat Neurosci, 7(12), 1345-1352. https://doi.org/10.1038/nn1352

Quilichini, P., Sirota, A., \& Buzsáki, G. (2010). Intrinsic circuit organization and theta-gamma oscillation dynamics in the entorhinal cortex of the rat. J Neurosci, 30(33), 11128-11142. https://doi.org/10.1523/JNEUROSCI.1327-10.2010

Quilichini, P. P., \& Bernard, C. (2012). Brain state-dependent neuronal computation. Front Comput Neurosci, 6, 77. https://doi.org/10.3389/fncom.2012.00077

Rissanen, J. (1978). Modeling by shortest data description. Automatica, 14(5), 465-471. https://doi.org/10.1016/0005-1098(78)90005-5

Rusina, E., Bernard, C., \& Williamson, A. (2021). Kainic Acid Models of Temporal Lobe Epilepsy. eNeuro.

Schneidman, E., Berry, M. J., 2nd, Segev, R., \& Bialek, W. (2006, Apr 20). Weak pairwise correlations imply strongly correlated network states in a neural population. Nature, 440(7087), 1007-1012. https://doi.org/10.1038/nature04701

Schreiber, T. (2000, Jul 10). Measuring information transfer. Phys Rev Lett, 85(2), 461-464. https://doi.org/10.1103/PhysRevLett.85.461

Scott, R. C., Menendez de la Prida, L., Mahoney, J. M., Kobow, K., Sankar, R., \& de Curtis, M. (2018, Aug). WONOEP APPRAISAL: The many facets of epilepsy networks. Epilepsia, 59(8), 1475-1483. https://doi.org/10.1111/epi.14503

Shannon, C. E. (1948). A Mathematical Theory of Communication. Bell System Technical Journal, 27(3), 379-423. https://doi.org/10.1002/j.1538-7305.1948.tb01338.x

Suarez, L. M., Cid, E., Gal, B., Inostroza, M., Brotons-Mas, J. R., Gomez-Dominguez, D., de la Prida, L. M., \& Solis, J. M. (2012). Systemic injection of kainic acid differently affects LTP magnitude depending on its epileptogenic efficiency. PLoS One, 7(10), e48128. https://doi.org/10.1371/journal.pone.0048128

Tatum, W. O. t. (2012, Oct). Mesial temporal lobe epilepsy. J Clin Neurophysiol, 29(5), 356-365. https://doi.org/10.1097/WNP.0b013e31826b3ab7 
916

917

918

919

920

921

922

923

924

925

926

927

928

929

930

931

932

933

934

935

936

937

938

939

940

941

942

943

944

945

946

947

948

949

Valero, M., Averkin, R. G., Fernandez-Lamo, I., Aguilar, J., Lopez-Pigozzi, D., Brotons-Mas, J. R., Cid, E., Tamas, G., \& Menendez de la Prida, L. (2017, Jun 21). Mechanisms for Selective Single-Cell Reactivation during Offline Sharp-Wave Ripples and Their Distortion by Fast Ripples. Neuron, 94(6), 1234-1247 e1237. https://doi.org/10.1016/i.neuron.2017.05.032

Van de Ville, D., Britz, J., \& Michel, C. M. (2010, Oct 19). EEG microstate sequences in healthy humans at rest reveal scale-free dynamics. Proc Natl Acad Sci U S A, 107(42), 18179-18184. https://doi.org/10.1073/pnas.1007841107

Wibral, M., Lizier, J. T., Vogler, S., Priesemann, V., \& Galuske, R. (2014). Local active information storage as a tool to understand distributed neural information processing. Front Neuroinform, 8, 1. https://doi.org/10.3389/fninf.2014.00001

Wibral, M., Priesemann, V., Kay, J. W., Lizier, J. T., \& Phillips, W. A. (2017, Mar). Partial information decomposition as a unified approach to the specification of neural goal functions. Brain Cogn, 112, 25-38. https://doi.org/10.1016/j.bandc.2015.09.004

Witter, M. P., Griffioen, A. W., Jorritsma-Byham, B., \& Krijnen, J. L. M. (1988). Entorhinal projections to the hippocampal CA1 region in the rat: An underestimated pathway. Neuroscience Letters, 85(2), 193-198. https://doi.org/10.1016/0304-3940(88)90350-3

Witter, M. P., Groenewegen, H. J., Lopes da Silva, F. H., \& Lohman, A. H. M. (1989). Functional organization of the extrinsic and intrinsic circuitry of the parahippocampal region. Progress in Neurobiology, 33(3), 161-253. https://doi.org/10.1016/0301-0082(89)90009-9

\section{Acknowledgements}

P.P.Q. and A.G. performed and administered all surgery, implantation, and experimental

recordings. P.P.Q, performed spike sorting, spectral analysis, and data pre-processing. W.C. and

D.B. performed computational analysis. T.M. assisted with computational analysis and many of

the supplementary materials. All authors designed the study and wrote the manuscript. W.C. is

funded through the M-GATE program, who has received funding from the European

Union's Horizon 2020 research and innovation program under the Marie Skłodowska-Curie

grant agreement No 765549. T.M was funded through Aix-Marseille Universite. P.P.Q.

acknowledges support from FRM, FFRE and CURE Epilepsy Taking Flight Award. D.B. has 
950 benefitted for this work from support provided by the French Agence Nationale pour la

951 Recherche (ERMUNDY, ANR-18-CE37-0014-02) and by the University of Strasbourg Institute for

952 Advanced Study (USIAS) for a Fellowship, within the French national programme "Investment

953 for the future" (IdEx-Unistra). C.B. is funded through ANR 19-CE14-0036-01. The funders had no

954 role in study design, data collection and analysis, decision to publish, or preparation of the

955 manuscript. The authors would like to thank Romain Goutagny and Anna Levina for scientific

956 discussions and comments regarding this work.

957

958 Data Availability

959 Partial data and codes can be found here: 10.5281/zenodo.4534369

960 Full codes, including figure generation as well as complete dataset are available upon request.

961

962 Competing Interests

963 The authors declare that they have no competing interests. 
$\mathbf{A}$
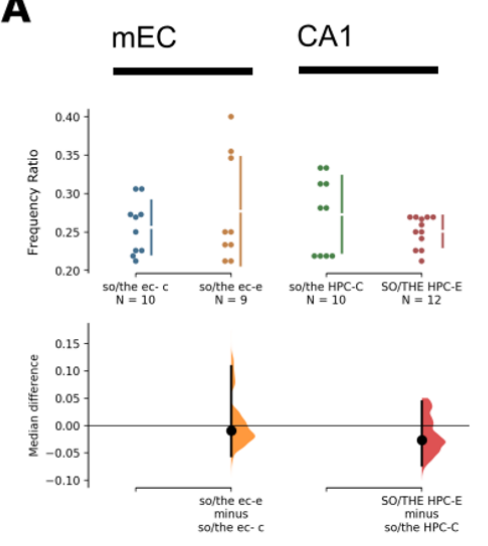

B

$\mathrm{mEC}$
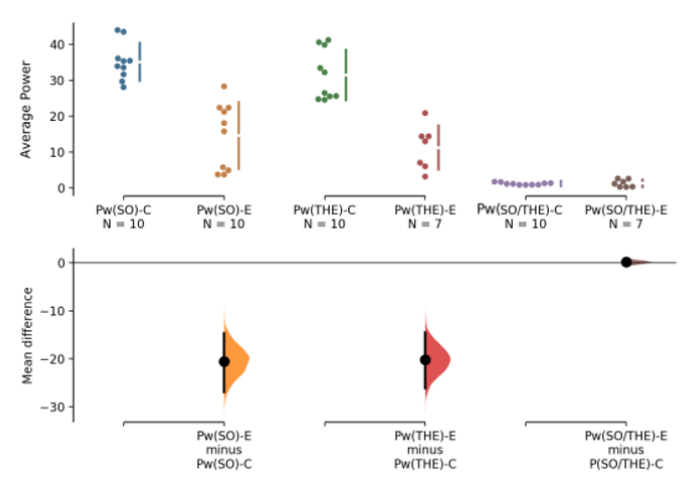

mEC
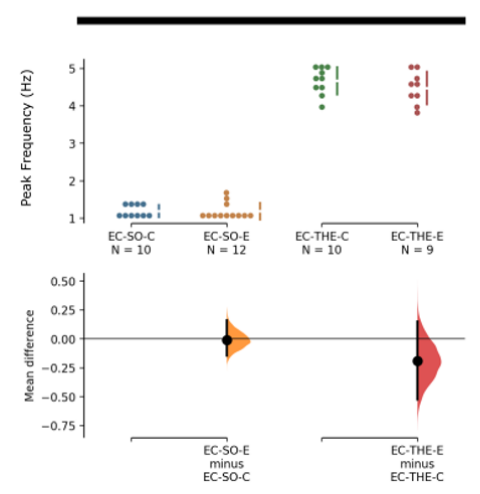

CA1
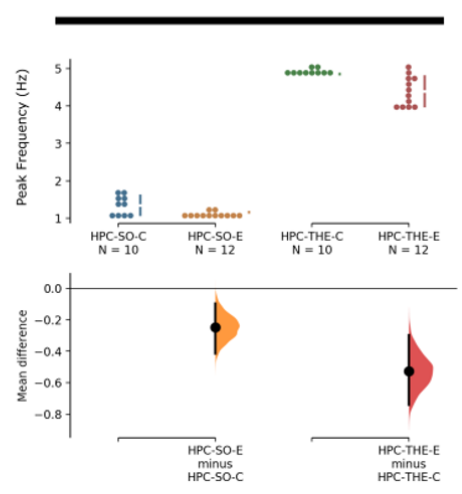

965

CA1
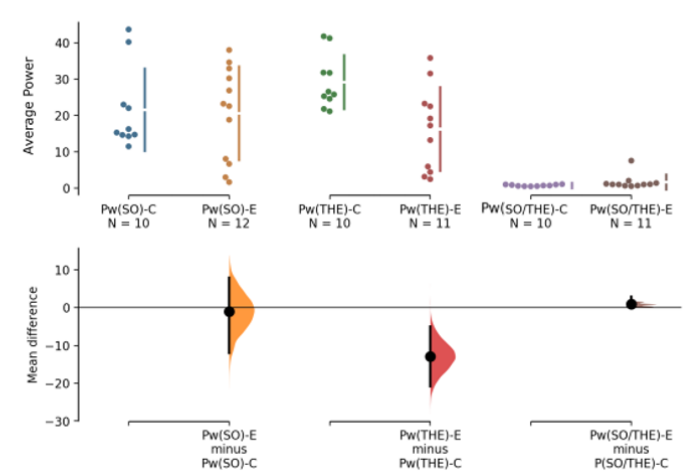

966 S1 - Frequency and Power Relationships in mEC and CA1 in control and epilepsy conditions -

967 (A) (Far Left) A comparison of ratios between peak frequencies during periods of SO and THE in

968 both control and epileptic conditions for mEC and CA1. (Middle and Right) Peak frequencies

969 used in the previous graph for periods of SO and THE in control and epilepsy for mEC and CA1.

970 There was a strong and smaller effect size on THE and SO peak frequency in CA1 in TLE,

971 respectively. In these Cumming estimation plots, circles represent the mean, and all bars

972 represent a 99\% bootstrapped confidence interval. (B) The average power found in periods of

973 SO and THE shown next to their ratio for both mEC and CA1. Note the strong effect size on THE 
bioRxiv preprint doi: https://doi.org/10.1101/2021.02.11.430768; this version posted February 12, 2021. The copyright holder for this preprint

(which was not certified by peer review) is the author/funder, who has granted bioRxiv a license to display the preprint in perpetuity. It is made available under aCC-BY-NC-ND 4.0 International license.

974 and SO power in the $\mathrm{mEC}$, and to a lesser extent on THE power in CA1. For all graphs, 5000

975 bootstrap samples were taken; the confidence interval is bias-corrected and accelerated. 

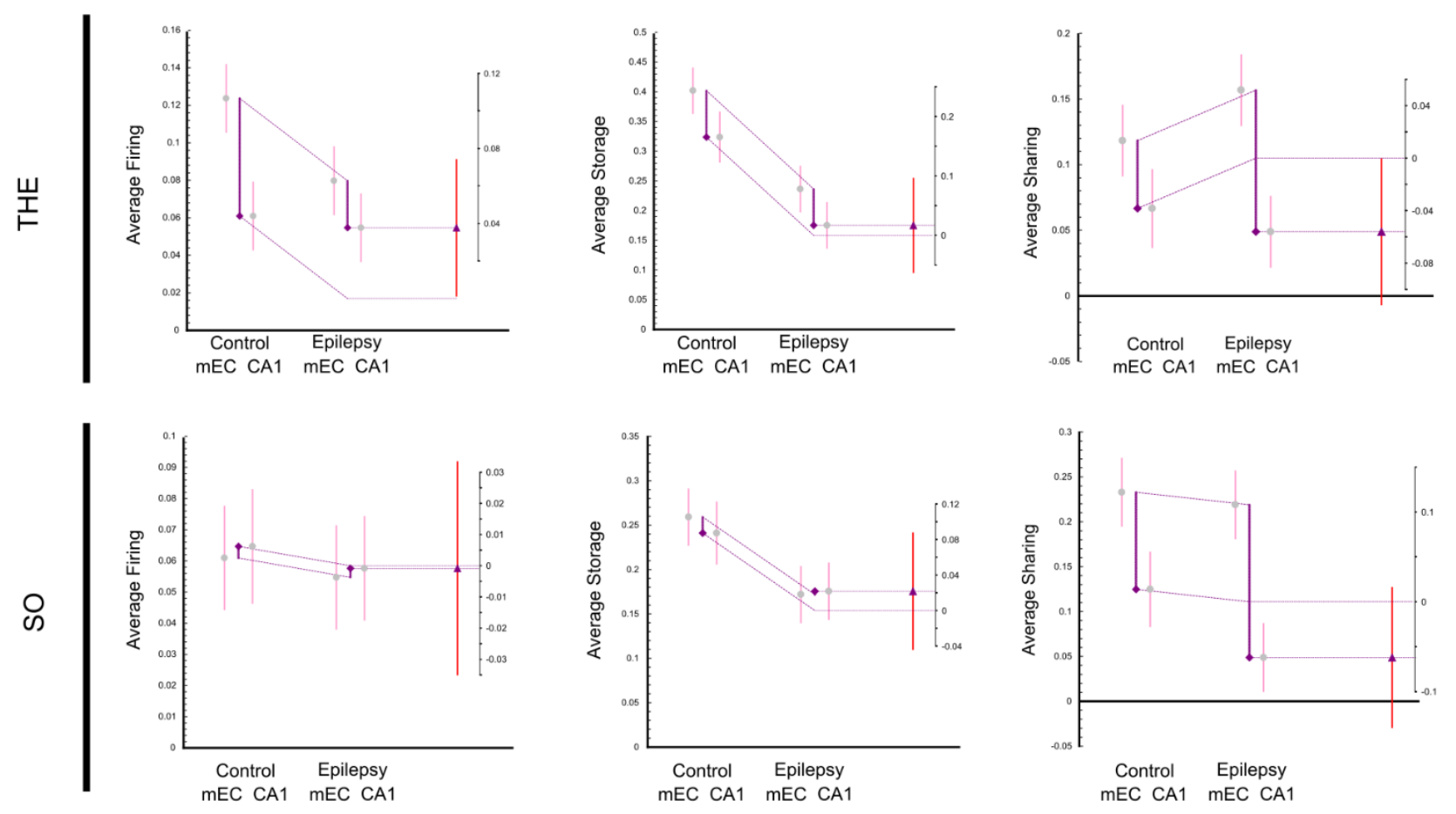

977 S2 - Average feature values presented as a function of $\mathrm{mEC}$ and CA1 in control and

978 epilepsy conditions - The same graph as Figure 1D presented in an alternate format to

979 highlight regional differences in control and epilepsy. The differences between mEC and

980 CA1 during THE and SO are similar in control and epilepsy for average firing and average

981 storage. The difference tends to increase for average sharing, but the effect size is

982 consistent Circles and triangles represent the mean, and all bars represent a 99\%

983 bootstrapped confidence interval. 


\section{Silhoutte Difference}
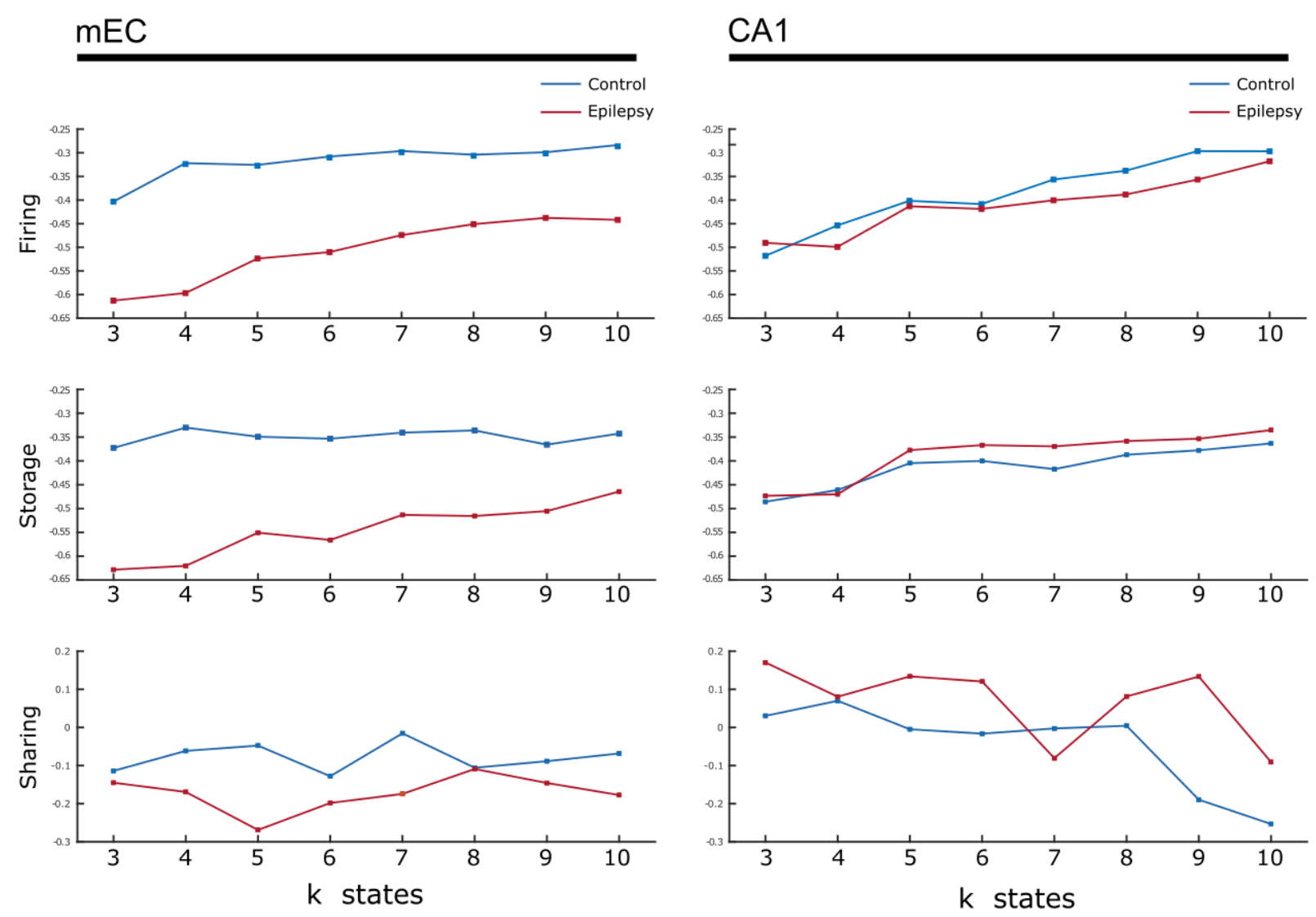

984

S3 - Null model with mean silhouette difference - The mean silhouette difference between a

randomized null clustering model and the silhouettes found using k-means on non-shuffled

987 data. Each point was calculated by computing mean silhouette values from a random selection

988 of the randomized and normal clustering and taking the difference. This was done 500 times to

989 produce error bars, but the error bars were so small that they appear to be squares on the

990 graph. The blue line is representative of control data and the red line represents epilepsy data.

991 There is a very large difference for firing and storage modalities from the null model for all $k$

992 values in both CA1 and $\mathrm{mEC}$ in control and epilepsy conditions. Of special note is the sharing

993 states found within CA1 (bottom right). We find that for both control and epilepsy conditions, 
994 our measure crosses 0 at $k=5$ and $k=7$, respectively, but fluctuates back above 0 until $k=9$ states

995 in control and k=10 in epilepsy. This would indicate that the clustering only weakly holds in

996 these intermediate values of $\mathrm{k}$ before not separating states better than a null, shuffled model

997 up until the higher $k$ values. Therefore, it may be that the states are either less definable in CA1

998 or, that on average there tend to be more states for sharing in both the control and epileptic

999 states in CA1 and would therefore require higher k, on average. 


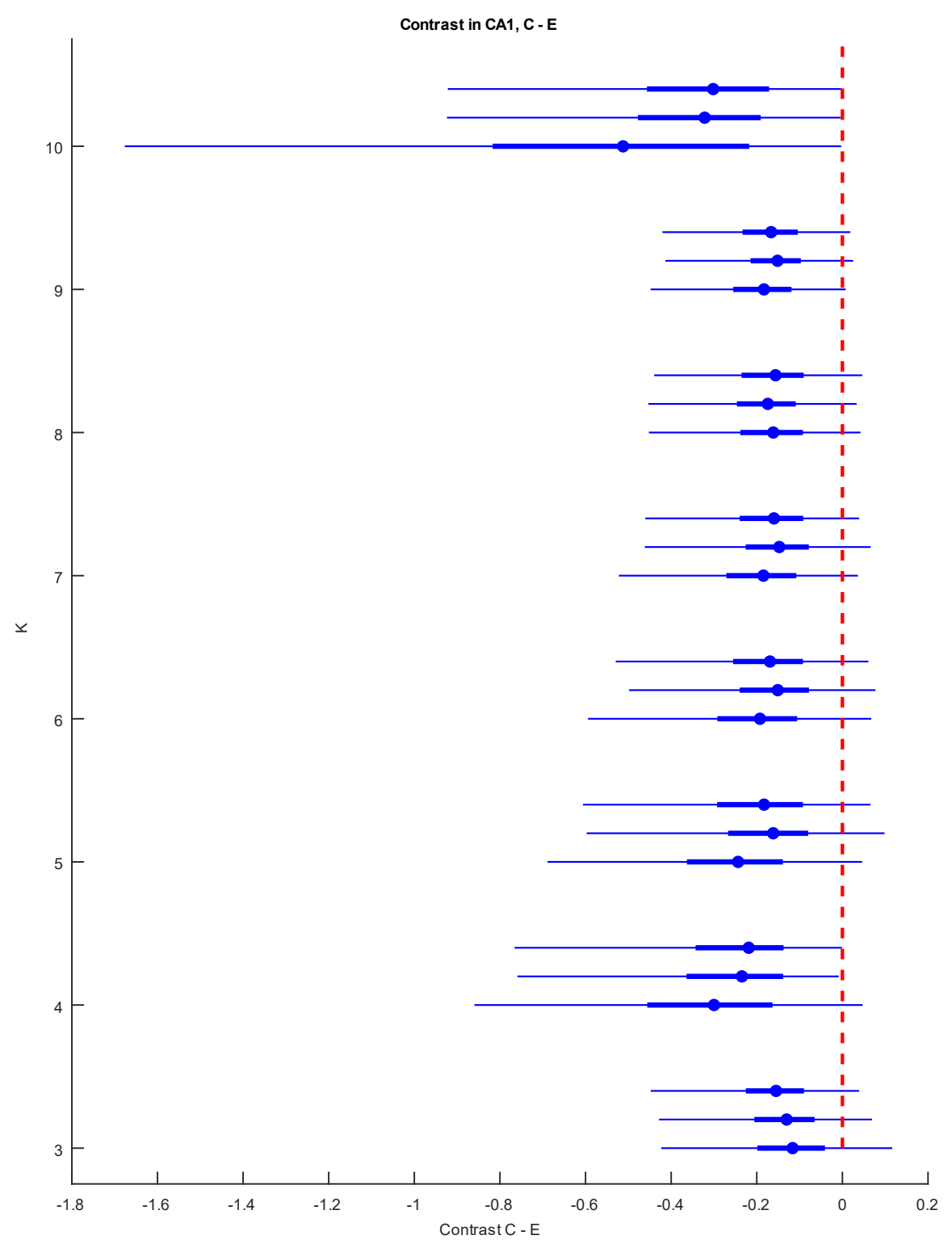

1000 S4 - Contrast Values for Control vs Epilepsy in CA1 - Average contrast difference between

1001 control and epilepsy is shown with respect to both feature and number of states, $k$. The circles

1002 represent the mean difference, the thick blue bars represent the $25-75 \%$ quantile and the thin

1003 blue bars represent the 1-99\% quantile. The red dotted line is to add the null hypothesis line of

1004 no significant difference between control and epilepsy. 
A
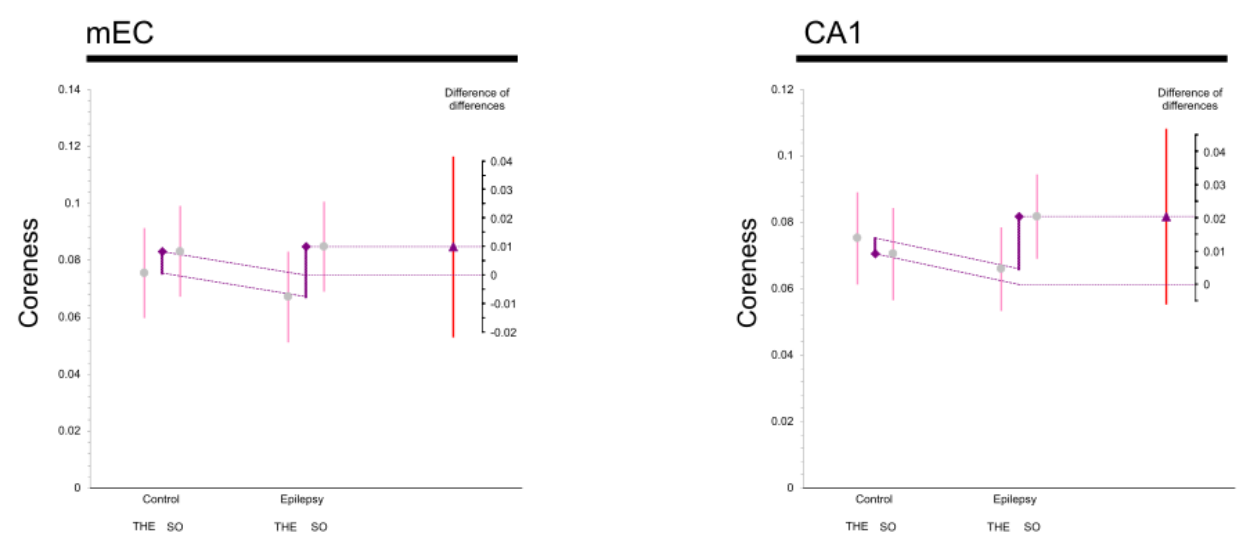

B
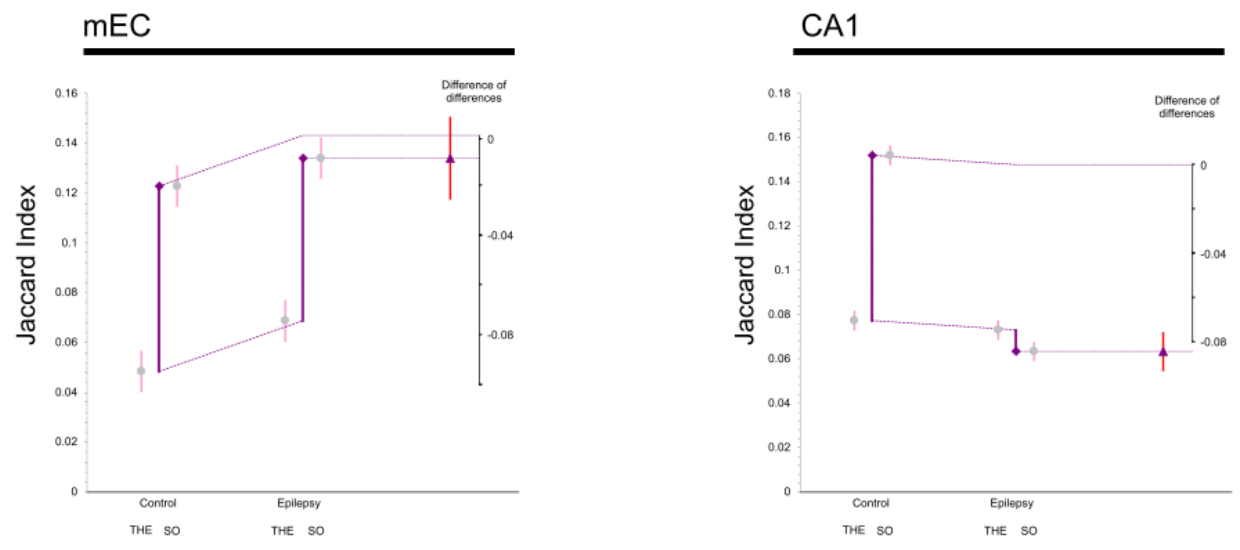

1006 S5 - Coreness and Jaccard Values - Average values and difference of differences graphs for

1007 data features taken from sharing networks, found using the sharing feature, for both

1008 control and epileptic animals. Circles and triangles represent the mean, and all bars

1009 represent a 99\% bootstrapped confidence interval. Note the very large effect size in the

1010 decrease of the Jaccard index in CA1 during SO. Accordingly, the brain state specificity of

1011 connectivity variance is lost. Significance is shown using the symbol $\left({ }^{*}\right)$ with their standard

1012 corresponding meaning $(*, p<0.05 ; * *, p<0.01 ; * * *, p<0.001)$. 
1013 ST1 - P value reporting: THE/SO unpaired mean difference

\begin{tabular}{|c|c|c|c|c|c|c|}
\hline & \multicolumn{3}{|c|}{$\mathrm{mEC}$} & \multicolumn{3}{c|}{ CA1 } \\
\hline Firing & $\begin{array}{c}\text { mean } \\
\text { difference }\end{array}$ & $\mathrm{Cl}$ & $\begin{array}{c}\mathrm{P} \\
\text { value }\end{array}$ & $\begin{array}{c}\text { mean } \\
\text { difference }\end{array}$ & $\mathrm{Cl}$ & P value \\
\hline Control & -0.0593 & $\begin{array}{c}{[-0.0815,-} \\
0.0365]\end{array}$ & 0 & -0.0475 & $\begin{array}{c}{[-0.0823,-} \\
0.0162]\end{array}$ & 0.0002 \\
\hline Epilepsy & -0.0217 & $\begin{array}{c}{[-0.0432,-} \\
2.55 e-05]\end{array}$ & 0.008 & 0.00082 & $\begin{array}{c}{[-0.0226,} \\
0.0263]\end{array}$ & 0.00082 \\
\hline Storage & -0.133 & $\begin{array}{c}{[-0.18,-} \\
0.0859]\end{array}$ & 0 & -0.0828 & $\begin{array}{c}{[-0.146,-} \\
0.0214]\end{array}$ & 0.0002 \\
\hline Control & -0.0584 & $\begin{array}{c}{[-0.0933,-} \\
0.0224]\end{array}$ & 0 & 0.0034 & $\begin{array}{c}{[-0.0452,} \\
0.0528]\end{array}$ & 0.0034 \\
\hline Epilepsy & 0.109 & $\begin{array}{c}{[0.0643,} \\
0.16]\end{array}$ & 0 & 0.0582 & $\begin{array}{c}0.0369, \\
0.0814]\end{array}$ & 0 \\
\hline Sharing & 0.0535 & $\begin{array}{c}{[-0.022,} \\
0.128]\end{array}$ & 0.0594 & 0.0285 & $\begin{array}{c}0.0105, \\
0.0435]\end{array}$ & 0 \\
\hline Control & & & & & \\
\hline Epilepsy & & & & & & \\
\hline
\end{tabular}

1014

1015 The $\mathrm{p}$-value reported here is from a two-sided permutation t-test with $\mathrm{Cl}$ intervals at $99 \% .5000$

1016 bootstrap samples were taken; the confidence interval is bias-corrected and accelerated. The $P$

1017 value(s) reported are the likelihood(s) of observing the effect size(s) if the null hypothesis of

1018 zero difference is true. For each permutation $P$ value, 5000 reshuffles of the control and test

1019 labels were performed. They are included here to satisfy a common requirement of scientific

1020 journals. (Ho et al., 2019) 
1021 ST2 - P value reporting: Difference of Difference Graphs

\begin{tabular}{|c|c|c|c|c|c|c|}
\hline & \multicolumn{3}{|c|}{$\mathrm{mEC}$} & \multicolumn{3}{|c|}{ HPC } \\
\hline & Effect & $\mathrm{Cl}$ & $\begin{array}{c}P \\
\text { value }\end{array}$ & Effect & $\mathrm{Cl}$ & $\begin{array}{c}P \\
\text { value }\end{array}$ \\
\hline \multicolumn{7}{|l|}{ Firing } \\
\hline Control v Epilepsy & -0.025 & {$[-0.043,-0.007]$} & $<0.001$ & -0.031 & $\begin{array}{c}{[-0.051,-} \\
0.011]\end{array}$ & $<0.001$ \\
\hline THE $\vee$ SO & -0.044 & {$[-0.062,-0.026]$} & $<0.001$ & -0.023 & $\begin{array}{c}{[-0.044,-} \\
0.003]\end{array}$ & 0.003 \\
\hline Diff of Diff & 0.0377 & {$[0.001,0.0744]$} & 0.008 & 0.048 & $\begin{array}{c}0.0072, \\
0.0889]\end{array}$ & 0.002 \\
\hline \multicolumn{7}{|l|}{ Storage } \\
\hline Control v Epilepsy & -0.126 & {$[-0.161,-0.092]$} & $<0.001$ & -0.107 & $\begin{array}{c}{[-0.147,-} \\
0.068]\end{array}$ & $<0.001$ \\
\hline THE v SO & -0.104 & {$[-0.138,-0.069]$} & $<0.001$ & -0.041 & $\begin{array}{c}{[-0.081,-} \\
0.002]\end{array}$ & 0.007 \\
\hline Diff of Diff & 0.0783 & {$[0.0099,0.1467]$} & 0.003 & 0.083 & $\begin{array}{c}0.0041 \\
0.1619]\end{array}$ & 0.007 \\
\hline \multicolumn{7}{|l|}{ Sharing } \\
\hline Control v Epilepsy & 0.0122 & {$[-0.032,0.0563]$} & 0.474 & -0.033 & $\begin{array}{c}-0.047,- \\
0.018]\end{array}$ & $<0.001$ \\
\hline THE v SO & 0.0844 & {$[0.0443,0.1325]$} & $<0.001$ & 0.0433 & $\begin{array}{l}\text { [0.029, } \\
0.0576]\end{array}$ & $<0.001$ \\
\hline Diff of Diff & -0.052 & {$[-0.141,0.0359]$} & 0.126 & -0.03 & {$[-0 .-58,-0.001]$} & 0.007 \\
\hline \multicolumn{7}{|l|}{ Coreness } \\
\hline Control v Epilepsy & -0.003 & {$[-0.019,0.0124]$} & 0.578 & 0.001 & $\begin{array}{l}{[-0.012} \\
0.0143]\end{array}$ & 0.849 \\
\hline THE v SO & 0.0126 & {$[-0.003,0.0285]$} & 0.04 & 0.0056 & $\begin{array}{l}{[-0.008} \\
0.0188] \\
\end{array}$ & 0.28 \\
\hline Diff of Diff & 0.0099 & {$[-0.022,0.0416]$} & 0.42 & 0.0205 & {$[-0.006,0.047]$} & 0.047 \\
\hline \multicolumn{7}{|l|}{ Jaccard } \\
\hline Control v Epilepsy & 0.0158 & {$[0.0074,0.0241]$} & $<0.001$ & -0.046 & $\begin{array}{c}{[-0.051,-} \\
0.042]\end{array}$ & $<0.001$ \\
\hline THE v SO & 0.0615 & {$[0.0615,0.0782]$} & $<0.001$ & 0.0325 & $\begin{array}{l}{[0.028,} \\
0.0369]\end{array}$ & $<0.001$ \\
\hline Diff of Diff & -0.009 & {$[-0.026,0.0076]$} & 0.16 & -0.084 & $\begin{array}{c}-0.093,- \\
0.075]\end{array}$ & $<0.001$ \\
\hline
\end{tabular}

